\title{
Neural circuitry underlying sustained attention in healthy adolescents and in ADHD symptomatology
}

Laura O'Halloran $^{\mathrm{a}}$, Zhipeng Cao ${ }^{\mathrm{b}}$, Kathy Ruddy ${ }^{\mathrm{a}}$, Lee Jollans ${ }^{\mathrm{a}}$, Matthew D. Albaugh ${ }^{\mathrm{c}}$, Andrea Aleni $^{\mathrm{a}}$, Alexandra S. Potter ${ }^{\mathrm{c}}$, Nigel Vahey ${ }^{\mathrm{a}}$, Tobias Banaschewski ${ }^{\mathrm{d}}$, Sarah Hohmann ${ }^{\mathrm{d}}$, Arun L.W. Bokde ${ }^{\mathrm{e}}$, Uli Bromberg ${ }^{\mathrm{f}}$, Christian Büchel $^{\mathrm{f}}$, Erin Burke Quinlan ${ }^{\mathrm{g}}$, Sylvane Desrivières ${ }^{g}$, Herta Flor $^{\mathrm{h}, \mathrm{i}}$, Vincent Frouin ${ }^{\mathrm{j}}$, Penny Gowland ${ }^{\mathrm{k}}$, Andreas Heinz ${ }^{1}$, Bernd Ittermann ${ }^{\mathrm{m}}$, Frauke Nees $^{\mathrm{d}, \mathrm{h}}$, Dimitri Papadopoulos Orfanos ${ }^{\mathrm{j}}$, Tomáš Paus ${ }^{\mathrm{n}}$, Michael N. Smolka ${ }^{\mathrm{o}}$, Henrik Walter ${ }^{1}$, Gunter Schumann ${ }^{g}$, Hugh Garavan ${ }^{c}$, Clare Kelly ${ }^{a}$, Robert Whelan ${ }^{\text {a,p, }}$

${ }^{a}$ School of Psychology and Institute of Neuroscience, Trinity College Dublin, Dublin 2, Ireland

${ }^{\mathrm{b}}$ School of Psychology, University College Dublin, Dublin 4, Ireland

${ }^{\mathrm{c}}$ Department of Psychiatry and Psychology, University of Vermont, 05405 Burlington, Vermont, USA

${ }^{\mathrm{d}}$ Department of Child and Adolescent Psychiatry and Psychotherapy, Central Institute of Mental Health, Medical Faculty Mannheim, Heidelberg University, Square J5, 68159 Mannheim, Germany

${ }^{\mathrm{e}}$ School of Medicine and Trinity College Institute of Neuroscience, Trinity College Dublin, Dublin, Ireland

${ }^{\mathrm{f}}$ University Medical Centre Hamburg-Eppendorf, House W34, 3.OG, Martinistr. 52, 20246, Hamburg, Germany

${ }^{\mathrm{g}}$ Medical Research Council, Social, Genetic and Developmental Psychiatry Centre, Institute of Psychiatry, Psychology \& Neuroscience, King's College London, United Kingdom

${ }^{\mathrm{h}}$ Department of Cognitive and Clinical Neuroscience, Central Institute of Mental Health, Medical Faculty Mannheim, Heidelberg University, Square J5, Mannheim, Germany

${ }^{i}$ Department of Psychology, School of Social Sciences, University of Mannheim, 68131 Mannheim, Germany

${ }^{\mathrm{j}}$ NeuroSpin, CEA, Université Paris-Saclay, F-91191 Gif-sur-Yvette, France

${ }^{\mathrm{k}}$ Sir Peter Mansfield Imaging Centre, School of Physics and Astronomy, University of Nottingham, University Park, Nottingham, United Kingdom

${ }^{1}$ Department of Psychiatry and Psychotherapy, Campus Charité Mitte, Charité, Universitätsmedizin Berlin, Charitéplatz 1, Berlin, Germany

${ }^{\mathrm{m}}$ Physikalisch-Technische Bundesanstalt (PTB), Braunschweig, Berlin, Germany

${ }^{\mathrm{n}}$ Rotman Research Institute, Baycrest and Departments of Psychology and Psychiatry, University of Toronto, Toronto, Ontario, M6A 2E1, Canada

${ }^{\circ}$ Department of Psychiatry and Neuroimaging Center, Technische Universität Dresden, Dresden, Germany

${ }^{\mathrm{p}}$ Global Brain Health Institute, Trinity College Dublin, Ireland

\section{A R T I C L E I N F O}

\section{Keywords:}

Functional connectivity

fMRI

Reaction-time variability

SST

Attention

ADHD

\section{A B S T R A C T}

Moment-to-moment reaction time variability on tasks of attention, often quantified by intra-individual response variability (IRV), provides a good indication of the degree to which an individual is vulnerable to lapses in sustained attention. Increased IRV is a hallmark of several disorders of attention, including Attention-Deficit/ Hyperactivity Disorder (ADHD). Here, task-based fMRI was used to provide the first examination of how average brain activation and functional connectivity patterns in adolescents are related to individual differences in sustained attention as measured by IRV. We computed IRV in a large sample of adolescents ( $\mathrm{n}=758)$ across 'Go' trials of a Stop Signal Task (SST). A data-driven, multi-step analysis approach was used to identify networks associated with low IRV (i.e., good sustained attention) and high IRV (i.e., poorer sustained attention). Low IRV was associated with greater functional segregation (i.e., stronger negative connectivity) amongst an array of brain networks, particularly between cerebellum and motor, cerebellum and prefrontal, and occipital and motor networks. In contrast, high IRV was associated with stronger positive connectivity within the motor network bilaterally and between motor and parietal, prefrontal, and limbic networks. Consistent with these observations, a separate sample of adolescents exhibiting elevated ADHD symptoms had increased fMRI activation and stronger positive connectivity within the same motor network denoting poorer sustained attention, compared to a matched asymptomatic control sample. With respect to the functional connectivity signature of low IRV, there were no statistically significant differences in networks denoting good sustained attention between the ADHD symptom

\footnotetext{
* Corresponding author. School of Psychology, Trinity College Dublin, Dublin 2, Ireland.

E-mail address: Robert.whelan@tcd.ie (R. Whelan).
} 
group and asymptomatic control group. We propose that sustained attentional processes are facilitated by an array of neural networks working together, and provide an empirical account of how the functional role of the cerebellum extends to cognition in adolescents. This work highlights the involvement of motor cortex in the integrity of sustained attention, and suggests that atypically strong connectivity within motor networks characterizes poor attentional capacity in both typically developing and ADHD symptomatic adolescents.

\section{Introduction}

The ability to efficiently and consistently maintain attentional resources on a moment-to-moment basis is central to our navigation of everyday life. Sustained attention can be examined behaviorally by measuring the intra-individual coefficient of variation (IRV), which examines within-person trial-to-trial reaction time (RT) inconsistency on a given cognitive task (Hultsch et al., 2002). IRV is particularly advantageous in that it is a relatively simple measurement that controls for overall speed of responding (e.g., it can be calculated as the standard deviation of RT divided by mean RT). IRV may provide a better metric of cognitive impairment than other neuropsychological test measures, such as standardized cognitive or psychomotor tasks (Haynes et al., 2017; Balota et al., 2010; Cherbuin et al., 2010) or simple RT (Dixon et al., 2007). Attentional deficits are commonly reported in attention deficit hyperactivity disorder (ADHD) during both laboratory tasks and in daily life (Klein et al., 2006; Leth-Steensen et al., 2000; Russell et al., 2006; Tamm et al., 2012; Barkley, 1997; Sonuga-Barke and Castellanos, 2007), with higher IRV commonly reported in ADHD (Kuntsi and Klein, 2011; Castellanos et al., 2006; Mullins et al., 2005; Kofler et al., 2013; Vaurio et al., 2009; Castellanos et al., 2005; Bellgrove et al., 2005).

\section{Brain correlates of sustained attention}

Neuroimaging studies have identified brain regions involved in sustained attention. For example, task-based fMRI analysis in 42 adults showed that high IRV (i.e., poorer sustained attention) was associated with activation in the middle frontal gyrus (MFG), motor (precentral gyrus and pre-supplementary area; SMA), parietal, thalamic and insula regions (Bellgrove et al., 2004). In healthy adults, low IRV (i.e., better sustained attention) was associated with stronger activation of anterior cingulate cortex (ACC) during a response inhibition task (Go/no-go task) (Johnson et al., 2015), and during a gradual onset continuous performance task (Esterman et al., 2012). In children (thirty 8-12-year-olds (Simmonds et al., 2007)), low IRV (i.e., better sustained attention) on a Go-No/Go task was associated with stronger Go activation in anterior cerebellum (culmen) and stronger No-Go activation in motor, frontoparietal (medial frontal gyrus; inferior parietal lobe, IPL) and cerebellar networks, while high IRV associated with stronger Go and No-Go activation in MFG, caudate and thalamus. To date, however, the brain correlates of sustained attention in healthy adolescents, as indexed by IRV, have not been comprehensively characterized. Furthermore, there has been a surge of interest not only in characterizing task-evoked regional activity, but also in discovering how such regions fit within large-scale neural networks in supporting sustained attention (Fortenbaugh et al., 2017).

Recent research has posited that sustained attentional processes may emerge from an array of large-scale functional connectivity networks (Castellanos et al., 2009; Kessler et al., 2016), rather than from single brain regions (Rosenberg et al., 2017; Chun et al., 2011). Functional connectivity - synchronous fluctuations in neural activity across the brain - can be measured by correlating the blood oxygenation leveldependent (BOLD) signal time course between two brain regions. The dorsal attention network (DAN; comprising intraparietal sulcus (IPS), superior parietal lobule; primate frontal eye fields, and inferior pre-central sulcus) and frontoparietal network have been established for their involvement in sustained attention (Petersen and Posner, 2012; Szczepanski et al., 2010). Stronger anticorrelations between task-positive networks and the default mode network (DMN; including medial prefrontal cortex, posterior cingulate, anterior temporal and precuneus) is associated lower IRV (Kelly et al., 2008). However, the extent to which other networks outside classic vigilance networks (e.g., cerebellum) contribute to sustaining attention is less well understood (Fortenbaugh et al., 2017; Glickstein, 2007). One study in particular (Rosenberg et al., 2015) examined the relationship between task-based functional connectivity and sustained attention (a measure of sensitivity called $d^{\prime}$ on a gradual-onset continuous performance task) in 25 healthy adults. They identified a low sustained attention network whose connectivity was associated with poorer sustained attention (low $d^{\prime}$ ), and a high sustained attention network whose connectivity was associated with better sustained attention (high $d^{\prime}$ ). The authors also tested the generalizability of these networks in comparison to separate resting-state data. Stronger connectivity between cerebellum with motor and occipital networks, and occipital with motor networks predicted better sustained attention. In contrast, stronger connectivity between temporal and parietal regions, and within the temporal lobe and cerebellum predicted poorer sustained attention, and also largely predicted ADHD symptom severity when applied to an independent sample of 113 8-16 year-olds with and without a diagnosis of ADHD. However, the $d^{\prime}$ measure used to assess sustained attention in this case likely captures a different facet of sustained attention than IRV. Moreover, examining commonalities in the brain networks implicated in sustained attention across different behavioral measures and datasets is an important step in elucidating the neural underpinning of individual differences in response variability.

\section{IRV and $A D H D$}

Functional connectivity in brain regions that have been previously implicated in poor attentional capacity in healthy (adult) individuals may also be disrupted in individuals with ADHD (Rosenberg et al., 2015). ADHD is associated with altered functional connectivity within and between the default, motor, cerebellar and frontoparietal networks (Castellanos et al., 2008; Barber et al., 2015), although findings in relation to functional connectivity and ADHD remain relatively heterogenous (Castellanos and Aoki, 2016). Neurological and psychopathological research is increasingly revealing a dimensionality aspect to developmental disorders such as ADHD (Hudziak et al., 2007) and conceptualizing attention-related traits as existing along a continuum shifts the focus from diagnostic groups towards diagnostic dimensions (O'Halloran et al., 2017). For example, reduced ventromedial prefrontal gray matter volume was associated with increased IRV in adolescents with elevated ADHD symptoms (Albaugh et al., 2017). Therefore, it is plausible that the (disrupted) functional connectivity patterns related to IRV in ADHD may be apparent among those with subclinical attention difficulties. This has yet to be examined.

\section{The present study}

In this study, we first sought to examine the relationship between fMRI activation and sustained attention, as measured by IRV on trials requiring a speeded response, in a large, normative sample of adolescents. This analysis identified a number of significant clusters, activation in which was then compared between a separate group of adolescents with ADHD symptoms and a matched asymptomatic control group. Next, given that sustained attention may be better characterized by the dynamic interactions of large scale brain networks than the degree of neural activation within single brain regions (Kuntsi and Klein, 2011; Mullins et al., 2005; Castellanos et al., 2005; Kelly et al., 2008; Glickstein, 2007), 
we examined the relationship between functional connectivity patterns and IRV in the normative sample. We computed a task-based functional connectivity matrix by correlating the BOLD signal time courses of every pair of regions in a 268-node brain atlas (Shen et al., 2013). This connectivity matrix was then correlated with each individual's IRV score, in order to identify networks associated with high and low IRV. Finally, we compared the IRV-linked networks identified in the normative sample between the ADHD symptom group and control group.

\section{Materials and methods}

\section{Participants}

Fourteen-year-olds were recruited at eight sites, and completed two fMRI sessions, psychiatric and neuropsychological assessments. Details of the full study protocol and data acquisition have been provided previously (Schumann et al., 2010) (http://www.imagen-europe.com/en/ Publications_and_SOP.php). Here, participants were allocated to one of three separate groups. The first was designated as the normative sample $(\mathrm{n}=758$; Table 1$)$. The second, the ADHD symptom sample $(\mathrm{n}=30$; Table 2), were selected according to the total score of ADHD parent ratings on the Development and Well Being Assessment (DAWBA; description below), with a threshold of two standard deviations higher than the mean ADHD score of the Imagen sample. A third group, the asymptomatic control sample $(\mathrm{n}=30$; Table 2$)$, had scores of 0 on the DAWBA for ADHD symptoms, and were matched for age, sex, recruitment sites, handedness, pubertal development, performance IQ and verbal IQ to the ADHD symptom group.

\section{Development and WellBeing assessment (DAWBA) interview}

The DAWBA (Goodman et al., 2000) is a structured set of questions designed to generate DSM-IV psychiatric diagnoses for children and adolescents aged 5-17 years. The ADHD subscale of the DAWBA consists of 31 questions, and includes specific ADHD subscales: hyperactive-impulsive, inattentive and combined. The DAWBA was administered to parents of the adolescents by questionnaire, under the supervision of a research assistant. Groups were constructed based on similar symptom cut-offs suggested by previous studies examining sub-clinical ADHD (Langley et al., 2012; Salum et al., 2014). The three subscales were added together to form an ADHD total score and the cut-off score for ADHD symptoms was calculated as two standard deviations from the mean total score, while a score of zero was required in order to classify a participant as a member of the control group (i.e. asymptomatic with respect to ADHD).

\section{Wechsler intelligence scale for children}

Participants completed a version of the Wechsler Intelligence Scale

Table 1

Summary statistics for the normative sample.

\begin{tabular}{ll}
\hline & Normative sample $(n=758)^{\mathrm{a}}$ \\
\hline Age (years) & $14.55(0.45)$ \\
Sex & 425 Females \\
Handedness & 664 Right \\
Pubertal Development & $3(0.69)$ \\
Performance IQ & $110(14)$ \\
Verbal IQ & $113(13)$ \\
IRV & $0.235(.038)$ \\
'Go' trial RT St. Dev. (ms) & $101(24)$ \\
'Go' trial mean RT (ms) & $429(61)$ \\
SSRT & $217(37)$ \\
Head Motion (Framewise displacement) & $0.212(.139)$ \\
Head Motion/IRV correlation & $.22^{\mathrm{b}}$ \\
\hline
\end{tabular}

\footnotetext{
a Mean (standard deviation), unless otherwise indicated.

b Spearman correlation, $p<.0001$.
}

for Children (WISC-IV) (Wechsler, 2003), which included the following subscales: Perceptual Reasoning, consisting of Block Design (arranging bi-colored blocks to duplicate a printed image) and Matrix Reasoning (the participant is presented with a series of colored matrices and must select the consistent pattern from a range of options); and Verbal Comprehension, consisting of Similarities (two similar but different objects or concepts are presented to the participant and they must explain how they are alike or different) and Vocabulary (a picture is presented or a word is spoken aloud by the experimenter and the participant is asked to provide the name of the depicted object or to define the word).

\section{Puberty development scale (PDS)}

The PDS scale (Petersen et al., 1988) assessed the pubertal status of the adolescent sample, by means of an eight-item self-report measure of physical development based on the Tanner stages, with separate forms for males and females. For this scale, there are five categories of pubertal status: (1) prepubertal, (2) beginning pubertal, (3) midpubertal, (4) advanced pubertal, (5) postpubertal. Participants answered questions about their growth in stature and pubic hair, as well as menarche in females and voice changes in males.

\section{Stop signal task}

Participants performed an adaptive event-related Stop Signal Task (SST) (Rubia et al., 2003, 2007), which took approximately $16 \mathrm{~min}$ to complete. The task consisted of 400 Go trials intermingled with 80 Stop trials; with between 3 and 7 Go trials between successive Stop trials. During Go trials participants were presented with arrows pointing either to the left or right, shown centrally on a screen for $1000 \mathrm{~ms}$. During Go trials participants were required to make a single button-press response with their left or right index finger corresponding to the direction of the arrow. In the unpredictable Stop trials, the arrows pointing left or right were followed (on average $300 \mathrm{~ms}$ later) by arrows pointing upwards (i.e. the Stop signal, shown for $100-300 \mathrm{~ms}$ ), which required participants to inhibit their motor responses during these trials. A tracking algorithm (Rubia et al., 2003, 2007) adjusted task difficulty by varying the stopsignal delay (SSD; the time interval between Go signal and Stop signal onsets; 250-900 ms in 50-ms increments), in accordance with each participant's performance on previous trials (average percentage of inhibition over previous Stop trials, recalculated after each Stop trial). The aim of this was to produce $50 \%$ successful and $50 \%$ unsuccessful inhibition trials. The inter-trial interval was jittered between 1.6 and $2.0 \mathrm{~s}$ (mean: $1.8 \mathrm{~s}$ ) to enhance statistical efficiency (Dale, 1999). If the participant

Table 2

Summary statistics for ADHD symptom and asymptomatic control groups.

\begin{tabular}{llll}
\hline & $\begin{array}{l}\text { ADHD } \\
(\mathrm{n}=30)\end{array}$ & $\begin{array}{l}\text { Control } \\
(\mathrm{n}=30)\end{array}$ & $p$ \\
\hline ADHD Total Score (DAWBA) & $43(9.83)$ & 0 & \\
Age & $14(0.38)$ & $14(0.41)$ & $.16^{\mathrm{a}}$ \\
Sex & 26 Males & 23 Males & $.32^{\mathrm{b}}$ \\
Handedness & 27 Right & 24 Right & $.28^{\mathrm{b}}$ \\
Pubertal Development & $3(0.50)$ & $3(0.71)$ & $.66^{\mathrm{c}}$ \\
Performance IQ & $101(13.06)$ & $103(15.11)$ & $.61^{\mathrm{a}}$ \\
Verbal IQ & $109(17.20)$ & $105(17.97)$ & $.48^{\mathrm{a}}$ \\
IRV & $0.258(0.04)$ & $0.228(0.36)$ & $<.005^{\mathrm{a}}$ \\
'Go' trial St. Dev. (ms) & $115(26.20)$ & $90(22.26)$ & $<.005^{\mathrm{a}}$ \\
'Go' trial mean RT (ms) & $446(72)$ & $391(58.56)$ & $<.005^{\mathrm{a}}$ \\
SSRT & $231(39)$ & $228(41)$ & $.76^{\mathrm{a}}$ \\
Head Motion (Framewise & 0.291 & 0.195 & $.03^{\mathrm{a}}$ \\
$\quad$ displacement) & $(0.218)$ & $(0.100)$ & \\
Head Motion/IRV correlation & $-.03^{\mathrm{d}}$ & $.08^{\mathrm{d}}$ & \\
\hline
\end{tabular}

a Two-sample two-tailed $t$-test.

b Chi-square test.

c Non-parametric Mann-Whitney $U$ test.

d Spearman correlation, $\mathrm{p}>.05$. 
responded to the Go stimulus before Stop stimulus presentation (i.e. stop too early; STE), then the trial was repeated (up to a maximum of seven trials).

We calculated each participants' Stop Signal RT (SSRT), an index of inhibitory function, by subtracting the mean stop-signal delay (SSD) from the Go RT at the percentile corresponding to the proportion of unsuccessful stop trials. In other words, the SSRT refers to the time taken to cancel a prepotent motor response after Stop stimulus presentation. IRV was calculated by dividing each individual's standard deviation of mean Go RT scores by their mean Go RT scores.

\section{MRI acquisition and analysis}

Functional MRI data were collected at eight IMAGEN sites (London, Nottingham, Dublin, Mannheim, Dresden, Berlin, Hamburg, and Paris) with 3T MRI systems made by various manufacturers (Siemens: 4 sites, Philips: 2 sites, General Electric: 1 site, and Bruker: 1 site). Standardized hardware for visual stimulus presentation (Nordic Neurolab, Bergen, Norway) was used at all sites. The MR scanning protocols, cross-site standardization and quality checks are further described in (Schumann et al., 2010). Functional runs included 444 whole-brain volumes acquired for each participant using echo-planar imaging (EPI) sequence. Each volume contained 40 axial slices aligned to the anterior commissure-posterior commissure (AC-PC) line (2.4-mm slice thickness, 1-mm slice gap). The echo time (TE) was optimized ( $\mathrm{TE}=30 \mathrm{~ms}$, repetition time $2200 \mathrm{~ms}$; flip angle $=75^{\circ}$; acquisition matrix $=64 \times 64$ ) to provide reliable imaging of subcortical areas.

\section{Preprocessing}

Preprocessing of the fMRI imaging data from IMAGEN was performed centrally using an automated pipeline with SPM8 (Statistical Parametric Mapping, (http://www.fil.ion.ucl.ac.uk/spm/)). fMRI BOLD images were co-registered with the T1W structural image (MPRAGE). Functional images were then realigned to correct for head motion and slice-time corrected using the first slice (top-down scanning) as reference for interpolation. T1W images were spatially normalized and non-linearly warped on Montreal Neurological Institute Coordinate System (MNI) space, using a custom EPI template. The custom template $(53 \times 63 \times 46$ voxels) was based on a subset of 240 participants' (30 from each of IMAGEN's eight sites) mean 480 EPI images that showed good spatial normalization, as measured by the overlap quality between individual EPI masks and the MNI mask (EPI images were spatially-realigned and their temporal-mean image was rigidly co-registered to their respective anatomical image). This normalization was applied to the EPI, and EPIs were then averaged to form an EPI template that was subsequently applied to all T1W data. Voxels were resampled at a resolution of $3 \times 3 \times 3 \mathrm{~mm}$. The functional data was then smoothed with a 4-mm full width half maximum Gaussian isotropic kernel. The contrast images were subsequently analyzed using SPM12 (http://www.fil.ion.ucl.ac.uk/spm/software/ spm12) and custom Matlab scripts (Mathworks).

\section{fMRI activation}

First-level activation maps were computed for go-trials, stop-success trials, and stop-fail trials versus baseline in individually specified general linear models (GLM). Design matrices included regressors for stopsuccess trials, stop-failure trials, Go too-late response trials, Go wrong response trials (i.e. wrong button press), movement parameters, and nuisance covariates (age, sex, pubertal status, handedness, performance IQ, verbal IQ, and data collection sites). On the second level, average fMRI activation for go-trials, stop-success trials, and stop-fail contrasts were each correlated with IRV for the normative sample using SPM12. Uncorrected $p$-values of .001 (recommended as the minimum lower limit (Eklund et al., 2017; Woo et al., 2014)), and a cluster extent of 32 contiguous voxels were used to provide a corrected family-wise error rate of $p<.05$. Significant clusters from each statistical parametric maps for the three contrasts were anatomically labelled by examining the MNI coordinates to xjview (http://www.alivelearn.net/xjview). Mean beta values from the significant clusters derived from the normative samples were extracted for the ADHD symptom group and asymptomatic control group. Between-group two-sample $t$-tests were performed to compare regions of interest (ROI) between groups. Bonferroni correction was applied based on the total number of ROIs.

\section{Task-based functional connectivity}

Whole-brain task-based functional connectivity was calculated using the following approach: We first removed the effect of Stop trials from the fMRI time series (using a similar principle to that described in (Fair et al., 2007a)). Specifically, we generated a general linear model (GLM) that included Stop-fail and stop-success trials and movement parameters. The Go condition ( $83 \%$ of trials) was not explicitly modelled. The residuals from this GLM, with stop-related activity and movement removed, were used in the task-based connectivity analysis. ROIs were derived from a 268-node functional brain atlas (referred to as the 'Shen atlas') that encompasses fine-grained, spatially homogeneous functional parcellations of the entire brain, including cortex, subcortical areas, and cerebellum, which serve as nodes for network analysis (Shen et al., 2013). Network labels, Brodmann areas (BA), and Montreal Neurological Institute (MNI) coordinates were automatically generated, and comprises ROIs with more coherent time courses than those defined by other atlases (e.g. automatic anatomic labeling atlas (Shen et al., 2013)). For each participant, the ROI timecourse was calculated by averaging the BOLD signal of all of its constituent voxels. This yielded $444 \times 268$ data points for each participant.

Since head motion occurs at low frequencies as intrinsic blood-oxygen level-dependent (BOLD) signal fluctuations, it can generate discrete neural artifacts that cannot be subjugated by increasing sample size or scan duration (Castellanos and Aoki, 2016). In order to further control for head motion artifacts, we included framewise displacement as a nuisance covariate in all connectivity analyses when computing partial correlations between functional connections and IRV (see below). Framewise displacement was defined as the sum of absolute scan to scan difference of the six translational and rotational realignment parameters (Power et al., 2014). We also conducted additional analyses to exclude head motion as a cause of spurious results: these analyses are described in Supplemental Information. The global signal (GS; average value across all gray-matter voxels) was included as a nuisance covariate once when computing the partial correlation between ROIs for each group (see below). The GS mitigates against between-subject effects of head motion (see (Fox et al., 2009; Yan et al., 2013)). Although GS regression can bias group differences by enhancing anti-correlated connections, and some caution should be taken when interpreting results (Saad et al., 2012), much of the variance in the global signal can be explained by head motion, respiratory noise, and scanner hardware-related artifacts (Power et al., 2017a).

A partial Pearson's correlation score was calculated among the 268 ROIs to determine their pairwise functional connectivity strength, with GS regressed as a nuisance covariate at this point. This yielded a connectivity matrix of size $268 \times 268$, with 35,778 unique connections between ROIs for each individual. Data file Supplemental_data_1. mat contains all pairwise correlations for all subjects. Matrices were not thresholded based on raw connection strength, allowing us to consider both low-variance connections (i.e., those that are consistently strongly positive or strongly negative across participants) and high-variance connections (i.e., those that are positive in some participants and negative in others); the latter, especially, may contain signal related to individual differences in IRV (see (Garrison et al., 2015; Scheinost et al., 2012)). 
Functional connectivity correlated with behavior

To assess the relevance of functional connections to behavior the following analysis was performed: The $268 \times 268$ matrix of connections between ROIs was correlated with each participant's IRV across the normative sample. Framewise displacement, age, sex, pubertal status, handedness, performance IQ, verbal IQ, and data collection site were nuisance covariate regressors. Type 1 error was estimated via randomlabel permutation by randomly shuffling IRV across participants and re-running the correlation analysis 1000 times in order to obtain an empirical null distribution. This analysis quantifies the probability of obtaining a particular $r$ value between IRV and functional connectivity by chance. The observed $r$ values between IRV and functional connectivity were considered significant if their associated $p$ value exceeded a particular percentile of the random-label permutation. The resulting thresholded matrix consisted of connections between ROIs that were negatively correlated with IRV (i.e., indexing good sustained attention) and connections between ROIs that were positively correlated with IRV (i.e., indexing poor sustained attention). This thresholding was repeated using a series of significance thresholds $(p<.001$, and $p<.0001)$ to identify networks associated with the task. Regional and network labels for the significant results were obtained from the previously available Shen atlas.

Having identified connections between ROIs that were significantly positively and negatively related to IRV using the $p<.001$ cutoff, (for comparison to similar research (Rosenberg et al., 2015)), we extracted and computed the same connections for the ADHD symptom and asymptomatic control groups. For each functional connection, the $r$-values were Fisher-normalized and then averaged across participants, within the ADHD symptom and asymptomatic control groups. This yielded two matrices for each group 1): connections positively correlated with IRV and 2) connections negatively correlated with IRV. Between-group two-sample $t$-tests were then conducted to examine group differences for each of these two connection types.

\section{Results}

Table 1 displays the summary characteristics of the normative sample and Table 2 displays the summary characteristics for the ADHD and control groups.

\section{Behavioral results}

The standard deviation of Go trial RT significantly correlated with the mean Go trial RT for the normative sample $(r=0.77, p<.001)$, the ADHD symptom group $(r=0.67, p<.001)$ and asymptomatic control group $(r=0.72, p<.001)$. The ADHD symptom group had significantly greater IRV $(M=0.258)$ than the matched asymptomatic control group $(M=0.228, \mathrm{t}(58)=-2.951, p=.005)$, and significantly greater IRV than the normative sample $(M=0.235, t(786)=-3.216, p=.001)$, while there was no significant difference in IRV between the normative sample and control group ( $\mathrm{t}(786)=-1.026, p=.305)$. SSRT was not significantly correlated with IRV for the normative sample $(r=0.06$, $p=.09$ ), the ADHD sample $(r=0.24, p=.19)$, or the control group $(r=-0.08, p=.66)$.

\section{fMRI activation results}

\section{Normative sample}

Whole-brain task activity (for Go trials, Stop Success and Stop Fail trials) significantly correlated with IRV in several brain areas in the normative sample (see Table 3 and Fig. 1). During Go trials, IRV was positively correlated with activation in bilateral postcentral gyrus, fusiform gyrus, superior temporal gyrus (STG), and right insula and precuneus. During Stop Fail trials, IRV was positively correlated with activation in left postcentral gyrus, and was negatively correlated with
Table 3

fMRI Activation correlated with IRV (Normative sample).

\begin{tabular}{|c|c|c|c|c|c|c|}
\hline \multirow[t]{2}{*}{$\begin{array}{l}\text { Brain Region } \\
\text { (direction of effect) }\end{array}$} & \multirow[t]{2}{*}{$\begin{array}{l}\text { Brodmann } \\
\text { Area }\end{array}$} & \multirow[t]{2}{*}{$\begin{array}{l}\text { Cluster } \\
\text { Size }\end{array}$} & \multirow[t]{2}{*}{$\begin{array}{l}\mathrm{Z} \\
\text { score }\end{array}$} & \multicolumn{3}{|c|}{$\begin{array}{l}\text { Montreal } \\
\text { Neurological } \\
\text { Institute (MNI) } \\
\text { Coordinates }\end{array}$} \\
\hline & & & & $\mathrm{x}$ & $\mathrm{y}$ & $\mathrm{z}$ \\
\hline \multicolumn{7}{|l|}{ Go trial (Positive) } \\
\hline Postcentral Gyrus R & & 280 & 4.564 & 60 & -28 & 46 \\
\hline Postcentral Gyrus L & & 405 & 5.083 & -45 & -25 & 64 \\
\hline Insula $\mathrm{R}$ & & 54 & 4.908 & 39 & -7 & 1 \\
\hline $\begin{array}{l}\text { Fusiform Gyrus } \\
\text { (Occipital) L }\end{array}$ & 18 & 47 & 4.903 & -21 & -76 & -14 \\
\hline $\begin{array}{l}\text { Fusiform Gyrus } \\
\text { (Occipital) R }\end{array}$ & & 41 & 4.884 & 21 & -34 & -20 \\
\hline $\begin{array}{l}\text { Lingual Gyrus } \\
\text { (Occipital) R }\end{array}$ & & 113 & 5.143 & 18 & -85 & -8 \\
\hline Precuneus R & & 39 & 4.713 & 27 & -70 & 37 \\
\hline STG L & 22 & 50 & 4.368 & -54 & -10 & 7 \\
\hline STG L & 41 & 47 & 4.237 & -45 & -25 & 7 \\
\hline & \multicolumn{5}{|c|}{ Stop Fail (Positive) } & 64 \\
\hline $\begin{array}{l}\text { Postcentral Gyrus L } \\
\text { Stop Fail (Negative) }\end{array}$ & 346 & 102 & 4.447 & -15 & -28 & 76 \\
\hline Insula L & 1347 & 105 & 5.062 & -36 & 14 & -2 \\
\hline Insula $\mathrm{R}$ & 1347 & 96 & 4.827 & 42 & 17 & -5 \\
\hline ACC R & 424 & 85 & 4.442 & 3 & 23 & 25 \\
\hline \multicolumn{7}{|c|}{ Stop Success (Positive) } \\
\hline Precentral Gyrus R & 46 & 98 & 5.418 & 27 & -25 & 76 \\
\hline Precentral Gyrus R & 46 & 84 & 5.200 & 54 & -7 & 52 \\
\hline Postcentral Gyrus L & 346 & 127 & 5.086 & -24 & -31 & 55 \\
\hline SMA L & 6 & 57 & 5.026 & 0 & -22 & 61 \\
\hline $\begin{array}{l}\text { Medial } \\
\quad \text { Orbitofrontal L }\end{array}$ & 10 & 45 & 4.698 & -6 & 62 & -5 \\
\hline Precuneus L & 31 & 176 & 4.499 & -12 & -55 & 16 \\
\hline Precuneus R & 23 & 46 & 4.465 & 18 & -58 & 19 \\
\hline Postcentral Gyrus L & 346 & 52 & 4.222 & -48 & -13 & 49 \\
\hline STG L & 226 & 37 & 3.898 & -60 & -16 & 4 \\
\hline \multicolumn{7}{|c|}{ Stop Success (Negative) } \\
\hline MFG R & 89 & 39 & 4.699 & 48 & 11 & 43 \\
\hline Insula $\mathrm{R}$ & 1347 & 52 & 4.675 & 45 & 17 & -5 \\
\hline Insula L & 1347 & 34 & 4.485 & -36 & 14 & -2 \\
\hline
\end{tabular}

*All regions survived corrections for multiple comparisons (FWE $p<.05$ ) at the whole brain cluster level. Abbreviations: $\mathrm{L}=\mathrm{Left}, \mathrm{R}=$ Right, $\mathrm{PCC}=$ Posterior Cingulate Cortex, $\mathrm{MOG}=$ Middle Occipital Gyrus, ACC $=$ Anterior Cingulate Cortex, SMA=Supplementary Motor Area, OFC $=$ Orbitofrontal cortex, STG Superior Temporal Gyrus, MFG $=$ Middle Frontal Gyrus.

activation in insula bilaterally and right anterior cingulate cortex (ACC). During Stop Success trials, IRV was positively correlated with activation in precentral gyrus bilaterally, left postcentral gyrus, right SMA, left medial orbitofrontal cortex (OFC), precuneus bilaterally, and left superior temporal gyrus (STG). During Stop Success trials, IRV was negatively correlated with activation in right MFG and insula bilaterally.

\section{ADHD symptom \& control groups}

Compared to the control group, the ADHD symptom group had significantly greater activation in left postcentral gyrus during Stop Fail trials (ADHD $m=0.30$, control $m=-0.20, p=.03$ ), during Stop Success trials (ADHD $m=0.12$, control $m=-0.27, p=.03$ ). No other significant differences emerged (using $p<.003$ the Bonferroni-corrected threshold for statistical significance).

\section{Functional connectivity results}

At the significance threshold of $p<.001$ (absolute $r$-value $>0.12$ derived from null models), 1368 connections between ROIs were associated with IRV. Networks linked with high and low IRV were identified (Fig. 2). The networks linked with high IRV (i.e., poor sustained attention) were primarily characterized by positive correlations between ROIs (610 connections between ROIs, $80 \%$ of which were positively correlated), while the networks linked with low IRV (i.e., good sustained 


\section{A. Go}
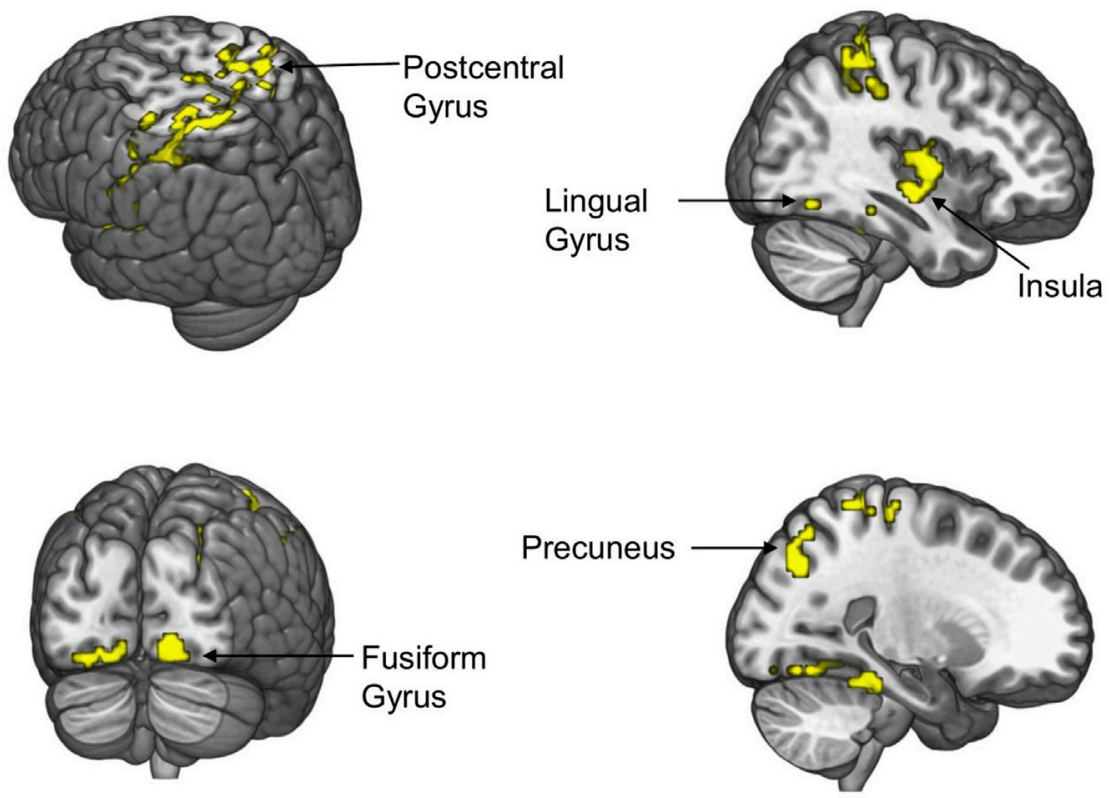

\section{B. Stop Fail}
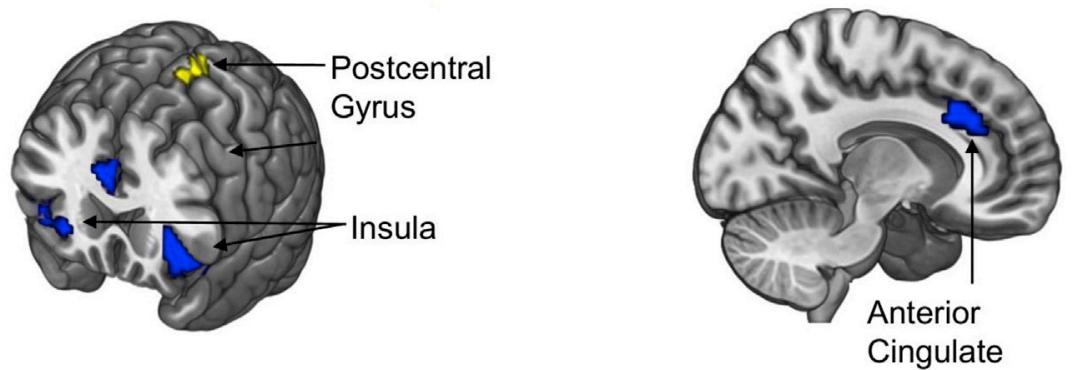

\section{Stop Success}
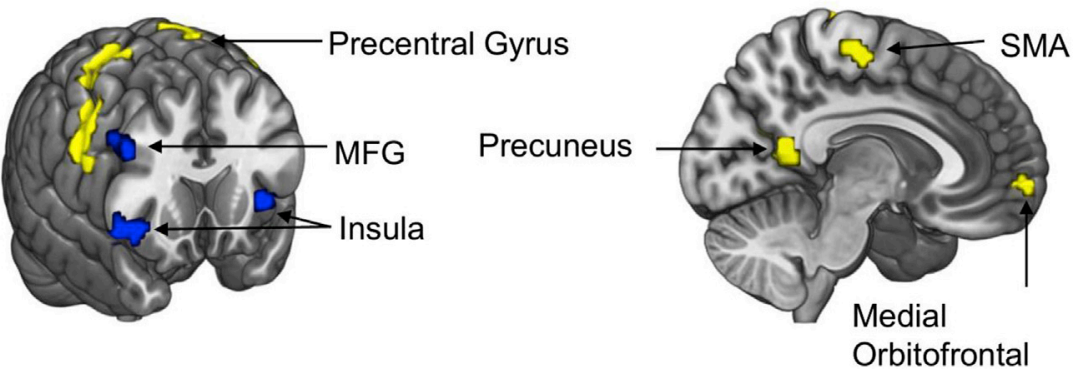

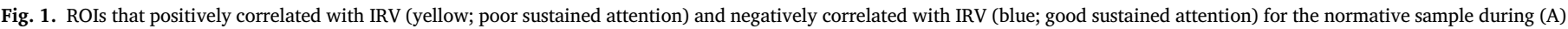
Go trials (B) Stop Fail and (C) Stop Success trials. Average fMRI activation images were created using MRIcroGL software (http://www.cabiatl.com/mricrogl/).

attention) were primarily characterized by negative correlations between ROIs (758 connections between ROIs, $86.7 \%$ of which were anticorrelated). In order to aid the interpretation of the findings (Böttger et al., 2014) the top connections between ROIs correlated with IRV are reported in Table 4, Fig. 2 \& Video 1 (full results contained in the Supplemental Data File 1 folder).

Supplementary video related to this article can be found at https:// doi.org/10.1016/j.neuroimage.2017.12.030.

Functional anatomy of attention networks

Network anatomy was intricate. However, several trends emerged (see Fig. 2). Connections positively correlated with IRV (i.e. poor sustained attention) were primarily located bilaterally within the motor network and between motor with parietal, prefrontal and limbic networks. The top 10 nodes positively correlated with IRV comprised positively correlated connections between ROIs between and within bilateral precentral and postcentral gyri. Connections negatively correlated with IRV (i.e. good sustained attention) were primarily negative (i.e., anti-correlated), indexing functional segregation between cerebellum with motor, prefrontal and parietal regions, and between occipital and motor networks. The top 10 connections between ROIs negatively correlated with IRV consisted of anti-correlations between left cerebellum crus I/II and right precentral/postcentral gyri.

ADHD symptom \& control groups

With respect to connections associated with high IRV (i.e., poor 


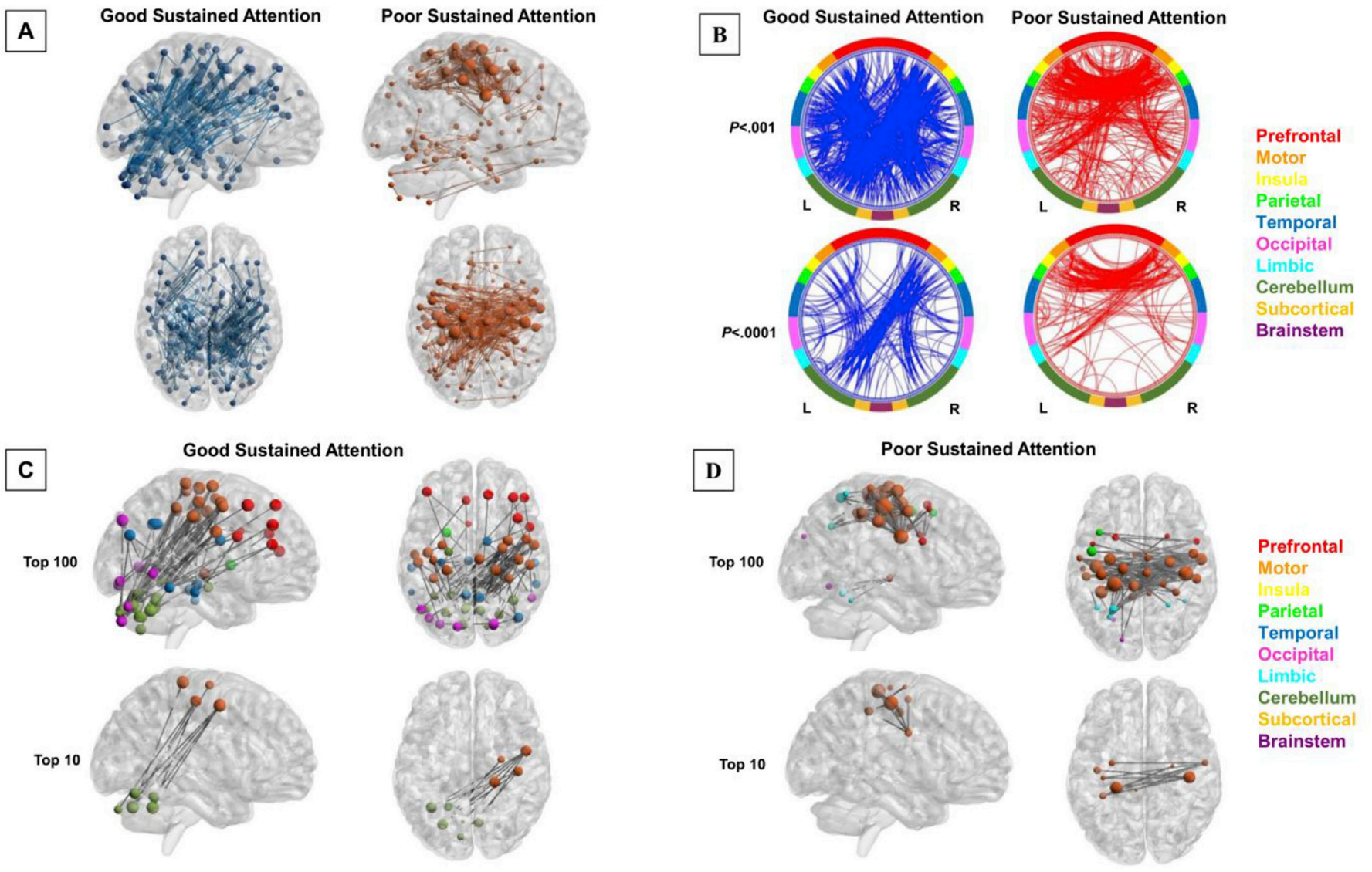

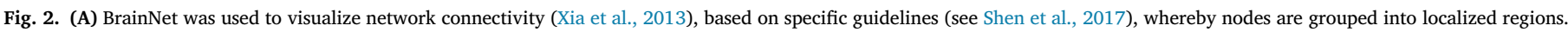

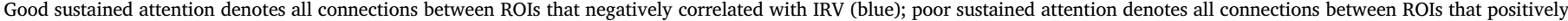

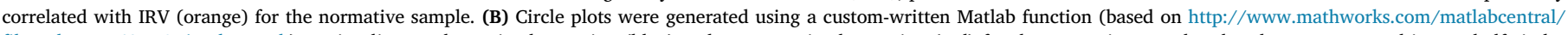

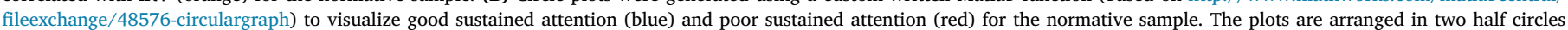

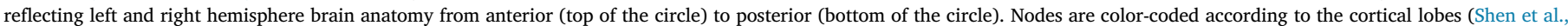

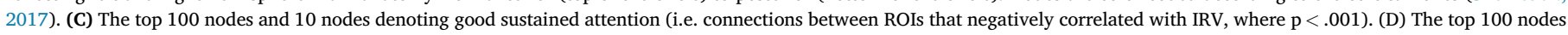

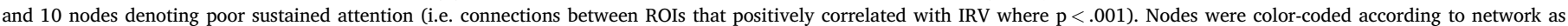
identified in (Xia et al., 2013).

sustained attention), the ADHD symptom exhibited significantly stronger positive connectivity between ROIs (Fisher-normalized $r$ value $=0.207$ ) than the control group (Fisher-normalized $r$ value $=0.156 t$ $(1218)=2.92, p=.003)$. There were no significant group differences in mean correlation strength for connections associated with low IRV (ADHD group, Fisher-normalized $r$ value $m=-0.132$; control group, Fisher-normalized $r$ value $m=-0.148, t(1514)=1.34, p=.177)$ See Fig. 3.

\section{Discussion}

To our knowledge, the current research is the first population-based functional imaging study to examine IRV with respect to both average fMRI activity and functional connectivity in a large cohort of adolescents, and in relation to ADHD symptomology. Average fMRI activation results indicated that good sustained attention (i.e., low IRV) was associated with increased bilateral activation in insula, ACC and prefrontal regions, while poor sustained attention (i.e., high IRV) was associated with increased bilateral activation in PCC, thalamus occipital and motor regions. The functional connectivity results indicated that good sustained attention was characterized by stronger negative connectivity (i.e., greater segregation) between cerebellum and motor networks, while stronger positive connectivity within the motor network was a signature of poorer sustained attention. Following this, we compared these sustained attention brain patterns in a separate sample of adolescents with ADHD symptoms to matched asymptomatic controls. Relative to controls, adolescents with ADHD symptoms had significantly higher IRV, increased Stop activation in postcentral gyrus, and stronger positive connectivity within low sustained attention networks associated with high IRV, as well as stronger positive connectivity within good sustained attention networks associated with low IRV. However, there were no significant differences between the groups for anti-correlated connections in networks associated with either high or low IRV.

For average fMRI activation, low IRV was associated with activation in right MFG and bilateral insula during Stop Success trials, and right ACC and insula bilaterally during Stop Fail trials. The ACC and insula cortices are part of the salience network, thought to be responsible for detecting behaviorally relevant cues and engaging executive processes (Namkung et al., 2017; Cai et al., 2014; Seeley et al., 2007; Wager and Barrett, 2017). With respect to the functional connectivity signature of low IRV, our findings highlight the importance of cerebellar network segregation in the brain. The top 10 nodes negatively correlated with IRV were all negative (left) cerebellar connections: with parietal lobe (right postcentral gyrus) and frontal areas (right SMA and dorsolateral prefrontal cortex; DLPFC), a finding that bears similarity in the adult connectivity literature (Rosenberg et al., 2015). The prominent task-active frontoparietal network, incorporating DLPFC, intraparietal sulcus and SMA, typically becomes more activated during attention-demanding tasks than during rest (Konrad and Eickhoff, 2010), and is associated with alertness, response preparation and selective attention (Sonuga-Barke and Castellanos, 2007; Fox et al., 2005). Prefrontal and parietal cortices have been implicated in numerous tasks of sustained attention 
Table 4

Top 30 connections between ROIs correlated with IRV.

\begin{tabular}{|c|c|c|c|c|c|c|c|c|c|c|c|c|c|c|c|c|}
\hline \multirow{2}{*}{$\begin{array}{l}\text { Brain Region } \\
1\end{array}$} & \multirow{2}{*}{$\begin{array}{l}\text { Brain Region } \\
2\end{array}$} & \multirow{2}{*}{$\begin{array}{l}\text { Hem } \\
1\end{array}$} & \multirow{2}{*}{$\begin{array}{l}\text { Hem } \\
2\end{array}$} & \multirow[t]{2}{*}{$\begin{array}{l}\text { BA } \\
1\end{array}$} & \multirow[t]{2}{*}{$\begin{array}{l}\text { BA } \\
2\end{array}$} & \multicolumn{3}{|l|}{$\begin{array}{l}\text { MNI } \\
1\end{array}$} & \multicolumn{3}{|l|}{$\begin{array}{l}\text { MNI } \\
2\end{array}$} & \multirow[b]{2}{*}{$\mathbf{p}$} & $\begin{array}{l}\text { Normative } \\
\text { FC \& IRV }\end{array}$ & FC & Control & ADHD \\
\hline & & & & & & $\mathbf{x}$ & $\mathbf{y}$ & $\mathbf{z}$ & $\mathbf{x}$ & $\mathbf{y}$ & $\mathbf{z}$ & & $r$ & $r$ & $r$ & $r$ \\
\hline High Sustained At & ntion & & & & & & & & & & & & & & & \\
\hline Postcentral Gyrus & Cerebellum Crus 1 & $\mathrm{R}$ & $\mathrm{L}$ & 2 & & 42 & -22 & 52 & -25 & -71 & -30 & .00 & -0.219 & -0.124 & 0.056 & 0.017 \\
\hline Precentral Gyrus & Cerebellum VI & $\mathrm{R}$ & $\mathrm{L}$ & 6 & & 49 & -3 & 49 & -7 & -68 & -18 & .00 & -0.216 & -0.373 & -0.311 & -0.548 \\
\hline Precentral Gyrus & Cerebellum Crus 1 & $\mathrm{R}$ & $\mathrm{L}$ & 6 & & 49 & -3 & 49 & -25 & -71 & -30 & .00 & -0.21 & -0.269 & -0.199 & -0.203 \\
\hline Postcentral Gyrus & Cerebellum Crus 2 & $\mathrm{R}$ & $\mathrm{L}$ & 2 & & 42 & -22 & 52 & -9 & -82 & -32 & .00 & -0.205 & -0.064 & 0.053 & -0.144 \\
\hline SMA & Cerebellum Crus 1 & $\mathrm{R}$ & $\mathrm{L}$ & 6 & & 27 & -11 & 65 & -25 & -71 & -30 & .00 & -0.204 & -0.09 & 0.089 & 0.064 \\
\hline Precentral Gyrus & Cerebellum VI & $\mathrm{R}$ & $\mathrm{L}$ & 6 & 38 & 49 & -3 & 49 & -20 & -55 & -22 & .00 & -0.2 & -0.361 & -0.244 & -0.437 \\
\hline Precentral Gyrus & Cerebellum VI & $\mathrm{R}$ & $\mathrm{R}$ & 6 & & 49 & -3 & 49 & 7 & -69 & -20 & .00 & -0.198 & -0.308 & -0.217 & -0.488 \\
\hline Postcentral Gyrus & Cerebellum Crus 1 & $\mathrm{R}$ & $\mathrm{L}$ & 2 & 37 & 42 & -22 & 52 & -35 & -55 & -31 & .00 & -0.197 & -0.163 & -0.09 & -0.017 \\
\hline Postcentral Gyrus & Cerebellum Crus 1 & $\mathrm{R}$ & $\mathrm{L}$ & 2 & & 21 & -32 & 67 & -25 & -71 & -30 & .00 & -0.193 & 0.052 & 0.292 & 0.241 \\
\hline Precentral Gyrus & Cerebellum Crus 1 & $\mathrm{R}$ & $\mathrm{L}$ & 6 & 37 & 49 & -3 & 49 & -35 & -55 & -31 & .00 & -0.193 & -0.29 & -0.274 & -0.256 \\
\hline Postcentral Gyrus & Cerebellum Crus 1 & $\mathrm{R}$ & $\mathrm{L}$ & 2 & & 42 & -22 & 52 & -25 & -71 & -30 & .00 & -0.191 & 0.000 & 0.202 & -0.038 \\
\hline Precentral Gyrus & Cerebellum Crus 2 & $\mathrm{R}$ & $\mathrm{L}$ & 6 & & 49 & -3 & 49 & -9 & -82 & -32 & .00 & -0.191 & -0.217 & -0.209 & -0.275 \\
\hline SMA & Cerebellum Crus 2 & $\mathrm{R}$ & $\mathrm{L}$ & 6 & & 27 & -11 & 65 & -9 & -82 & -32 & .00 & -0.189 & -0.053 & 0.014 & -0.08 \\
\hline DLPFC & MTG & $\mathrm{R}$ & $\mathrm{R}$ & 46 & 37 & 47 & 35 & 19 & 59 & -45 & -15 & .00 & -0.188 & -0.412 & -0.617 & -0.352 \\
\hline Postcentral Gyrus & Cerebellum VI & $\mathrm{R}$ & $\mathrm{L}$ & 2 & & 42 & -22 & 52 & -7 & -68 & -18 & .00 & -0.187 & -0.269 & -0.153 & -0.386 \\
\hline Precentral Gyrus & Cerebellum V & $\mathrm{R}$ & $\mathrm{L}$ & 6 & & 49 & -3 & 49 & -6 & -56 & -25 & .00 & -0.185 & -0.359 & -0.267 & -0.506 \\
\hline Postcentral Gyrus & Cerebellum Crus 2 & $\mathrm{R}$ & $\mathrm{L}$ & 40 & & 53 & -27 & 41 & -9 & -82 & -32 & .00 & -0.183 & -0.147 & -0.071 & -0.12 \\
\hline Postcentral Gyrus & Cerebellum VI & $\mathrm{R}$ & $\mathrm{R}$ & 2 & & 42 & -22 & 52 & 24 & -73 & -28 & .00 & -0.183 & -0.018 & 0.153 & -0.016 \\
\hline Postcentral Gyrus & Cerebellum Crus 1 & $\mathrm{R}$ & $\mathrm{L}$ & 40 & & 53 & -27 & 41 & -25 & -71 & -30 & .00 & -0.181 & -0.235 & -0.09 & 0.000 \\
\hline Postcentral Gyrus & Cerebellum Crus 1 & $\mathrm{~L}$ & $\mathrm{~L}$ & 1 & & -36 & -23 & 64 & -25 & -71 & -30 & .00 & -0.179 & -0.026 & 0.222 & 0.014 \\
\hline SMA & Cerebellum Crus 1 & $\mathrm{R}$ & $\mathrm{L}$ & 6 & 37 & 27 & -11 & 65 & -35 & -55 & -31 & .00 & -0.179 & -0.119 & -0.052 & -0.04 \\
\hline SMA & Cerebellum VI & $\mathrm{R}$ & $\mathrm{L}$ & 6 & & 27 & -11 & 65 & -7 & -68 & -18 & .00 & -0.178 & -0.287 & -0.254 & -0.398 \\
\hline Postcentral Gyrus & Cerebellum Crus 2 & $\mathrm{R}$ & $\mathrm{L}$ & 2 & & 21 & -32 & 67 & -9 & -82 & -32 & .00 & -0.178 & 0.06 & 0.176 & 0.009 \\
\hline IPL & Cerebellum Crus 2 & $\mathrm{R}$ & $\mathrm{L}$ & 2 & & 33 & -39 & 48 & -9 & -82 & -32 & .00 & -0.176 & -0.02 & 0.003 & -0.029 \\
\hline SMA & Cerebellum VI & $\mathrm{R}$ & $\mathrm{R}$ & 6 & & 27 & -11 & 65 & 24 & -73 & -28 & .00 & -0.176 & -0.011 & 0.111 & 0.097 \\
\hline MTG & DLPFC & $\mathrm{R}$ & $\mathrm{L}$ & 37 & 46 & 59 & -45 & -15 & -42 & 41 & 14 & .00 & -0.174 & -0.372 & -0.573 & -0.314 \\
\hline IPL & Cerebellum Crus 1 & $\mathrm{R}$ & $\mathrm{L}$ & 2 & & 33 & -39 & 48 & -25 & -71 & -30 & .00 & -0.174 & -0.091 & 0.067 & 0.101 \\
\hline Postcentral Gyrus & Cerebellum Crus 2 & $\mathrm{~L}$ & $\mathrm{~L}$ & 1 & & -36 & -23 & 64 & -9 & -82 & -32 & .00 & -0.172 & -0.052 & 0.167 & -0.248 \\
\hline SFG & MTG & $\mathrm{R}$ & $\mathrm{R}$ & 10 & 37 & 37 & 36 & 35 & 59 & -45 & -15 & .00 & -0.172 & -0.437 & -0.545 & -0.308 \\
\hline Postcentral Gyrus & Cerebellum VI & $\mathrm{R}$ & $\mathrm{L}$ & 40 & & 53 & -27 & 41 & -7 & -68 & -18 & .00 & -0.171 & -0.294 & -0.165 & -0.333 \\
\hline Low Sustained Att & tion & & & & & & & & & & & & & & & \\
\hline Precentral Gyrus & $\overline{\text { Postcentral Gyrus }}$ & $\mathrm{R}$ & $\mathrm{L}$ & 2 & 1 & 42 & -22 & 52 & -24 & -32 & 61 & .00 & 0.243 & 0.307 & 0.318 & 0.579 \\
\hline Postcentral Gyrus & Postcentral Gyrus & $\mathrm{R}$ & $\mathrm{L}$ & 2 & 4 & 42 & -22 & 52 & -41 & -16 & 45 & .00 & 0.227 & 0.206 & 0.02 & 0.047 \\
\hline Postcentral Gyrus & IPL & $\mathrm{R}$ & $\mathrm{L}$ & 2 & 40 & 42 & -22 & 52 & -36 & -39 & 46 & .00 & 0.226 & 0.17 & 0.06 & 0.425 \\
\hline Precentral Gyrus & Postcentral Gyrus & $\mathrm{R}$ & $\mathrm{L}$ & 4 & 1 & 57 & -9 & 29 & -24 & -32 & 61 & .00 & 0.223 & -0.152 & -0.261 & -0.033 \\
\hline Precentral Gyrus & Claustrum & $\mathrm{R}$ & $\mathrm{L}$ & 4 & 7 & 57 & -9 & 29 & -28 & -9 & 55 & .00 & 0.221 & 0.053 & -0.132 & -0.048 \\
\hline Precentral Gyrus & Postcentral Gyrus & $\mathrm{R}$ & $\mathrm{L}$ & 4 & 1 & 57 & -9 & 29 & -36 & -23 & 64 & .00 & 0.216 & -0.178 & -0.441 & 0.002 \\
\hline Precentral Gyrus & IPL & $\mathrm{R}$ & $\mathrm{L}$ & 4 & 40 & 57 & -9 & 29 & -36 & -39 & 46 & .00 & 0.215 & -0.067 & -0.318 & 0.026 \\
\hline MFG & IPL & $\mathrm{R}$ & $\mathrm{L}$ & 6 & 40 & 27 & -11 & 65 & -36 & -39 & 46 & .00 & 0.212 & 0.19 & 0.22 & 0.393 \\
\hline Postcentral Gyrus & Postcentral Gyrus & $\mathrm{R}$ & $\mathrm{L}$ & 2 & 1 & 42 & -22 & 52 & -36 & -23 & 64 & .00 & 0.212 & 0.294 & 0.235 & 0.413 \\
\hline Precentral Gyrus & Precuneus & $\mathrm{R}$ & $\mathrm{L}$ & 4 & 8 & 57 & -9 & 29 & -6 & -34 & 64 & .00 & 0.211 & -0.073 & -0.171 & 0.032 \\
\hline Postcentral Gyrus & SFG & $\mathrm{R}$ & $\mathrm{L}$ & 2 & 7 & 42 & -22 & 52 & -16 & -18 & 68 & .00 & 0.21 & 0.338 & 0.52 & 0.591 \\
\hline Precentral Gyrus & Postcentral Gyrus & $\mathrm{R}$ & $\mathrm{L}$ & 6 & 1 & 49 & -3 & 49 & -36 & -23 & 64 & .00 & 0.204 & 0.161 & -0.004 & 0.379 \\
\hline Precentral Gyrus & Insula & $\mathrm{R}$ & $\mathrm{L}$ & 4 & 6 & 57 & -9 & 29 & -45 & -1 & 49 & .00 & 0.204 & -0.049 & -0.276 & -0.161 \\
\hline Postcentral Gyrus & Postcentral Gyrus & $\mathrm{R}$ & $\mathrm{L}$ & 40 & 1 & 53 & -27 & 41 & -24 & -32 & 61 & .00 & 0.204 & 0.198 & 0.188 & 0.552 \\
\hline Precentral Gyrus & Postcentral Gyrus & $\mathrm{R}$ & $\mathrm{L}$ & 6 & 1 & 49 & -3 & 49 & -24 & -32 & 61 & .00 & 0.201 & 0.108 & 0.012 & 0.407 \\
\hline MFG & Postcentral Gyrus & $\mathrm{R}$ & $\mathrm{L}$ & 6 & 1 & 27 & -11 & 65 & -24 & -32 & 61 & .00 & 0.2 & 0.293 & 0.286 & 0.391 \\
\hline Postcentral Gyrus & SPL & $\mathrm{R}$ & $\mathrm{L}$ & 2 & 1 & 42 & -22 & 52 & -51 & -25 & 40 & .00 & 0.198 & 0.084 & -0.027 & 0.12 \\
\hline Precentral Gyrus & SMA & $\mathrm{R}$ & $\mathrm{R}$ & 4 & 6 & 57 & -9 & 29 & 6 & -22 & 63 & .00 & 0.198 & -0.043 & -0.117 & 0.013 \\
\hline MFG & Postcentral Gyrus & $\mathrm{R}$ & $\mathrm{L}$ & 6 & 1 & 27 & -11 & 65 & -36 & -23 & 64 & .00 & 0.197 & 0.354 & 0.381 & 0.409 \\
\hline MFG & Postcentral Gyrus & $\mathrm{R}$ & $\mathrm{L}$ & 6 & 4 & 27 & -11 & 65 & -41 & -16 & 45 & .00 & 0.196 & 0.281 & 0.228 & 0.151 \\
\hline Postcentral Gyrus & Precuneus & $\mathrm{R}$ & $\mathrm{L}$ & 2 & 8 & 42 & -22 & 52 & -6 & -34 & 64 & .00 & 0.195 & 0.407 & 0.516 & 0.718 \\
\hline Precentral Gyrus & Postcentral Gyrus & $\mathrm{R}$ & $\mathrm{R}$ & 4 & 2 & 57 & -9 & 29 & 42 & -22 & 52 & .00 & 0.195 & 0.022 & -0.346 & -0.135 \\
\hline MFG & IPL & $\mathrm{R}$ & $\mathrm{L}$ & 6 & 7 & 27 & -11 & 65 & -25 & -55 & 59 & .00 & 0.193 & 0.224 & 0.329 & 0.44 \\
\hline Precentral Gyrus & Postcentral Gyrus & $\mathrm{R}$ & $\mathrm{L}$ & 4 & 4 & 57 & -9 & 29 & -41 & -16 & 45 & .00 & 0.192 & -0.14 & -0.453 & -0.113 \\
\hline IPL & Postcentral Gyrus & $\mathrm{R}$ & $\mathrm{L}$ & 2 & 1 & 33 & -39 & 48 & -24 & -32 & 61 & .00 & 0.189 & 0.309 & 0.389 & 0.466 \\
\hline Postcentral Gyrus & SFG & $\mathrm{R}$ & $\mathrm{L}$ & 40 & 7 & 53 & -27 & 41 & -16 & -18 & 68 & .00 & 0.187 & 0.245 & 0.375 & 0.493 \\
\hline MFG & SPL & $\mathrm{R}$ & $\mathrm{L}$ & 6 & 1 & 27 & -11 & 65 & -51 & -25 & 40 & .00 & 0.187 & 0.141 & 0.175 & 0.245 \\
\hline Precentral Gyrus & Insula & $\mathrm{R}$ & $\mathrm{L}$ & 4 & 7 & 57 & -9 & 29 & -23 & 12 & 54 & .00 & 0.184 & 0.27 & 0.155 & -0.011 \\
\hline Postcentral Gyrus & Postcentral Gyrus & $\mathrm{R}$ & $\mathrm{L}$ & 40 & 4 & 53 & -27 & 41 & -41 & -16 & 45 & .00 & 0.183 & 0.093 & -0.174 & -0.215 \\
\hline Postcentral Gyrus & Precuneus & $\mathrm{R}$ & $\mathrm{L}$ & 40 & 8 & 53 & -27 & 41 & -6 & -34 & 64 & .00 & 0.182 & 0.347 & 0.428 & 0.722 \\
\hline
\end{tabular}

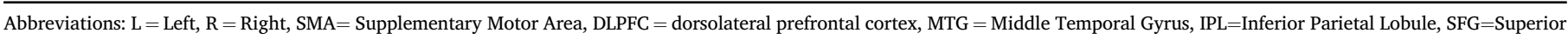
Frontal Gyrus, MFG = Middle Frontal Gyrus, SPL=Superior Parietal Lobule.

(Langner and Eickhoff, 2013; Corbetta and Shulman, 2002) and these findings lend support to previous structural findings, which linked prefrontal anomalies to increased IRV (Albaugh et al., 2017).

The cerebellum is thought to have a critical role in sustained attention (Habas et al., 2009; Stoodley, 2012; Buckner, 2013). In healthy adults, recent work has shown that enhancing cerebellar functional connectivity via transcranial magnetic stimulation can decrease IRV (Esterman et al., 2017). Distinct subregions of the cerebellum have been identified as being coupled with specific cerebral networks (Brisenden et al., 2016; Buckner et al., 2011). For example, positive connectivity between right hemispheric cerebellar lobules VIIbIVIIIa with DAN were robustly recruited in a series of resting-state and working memory/sustained 


\section{ADHD > Controls Poor Sustained Attention}
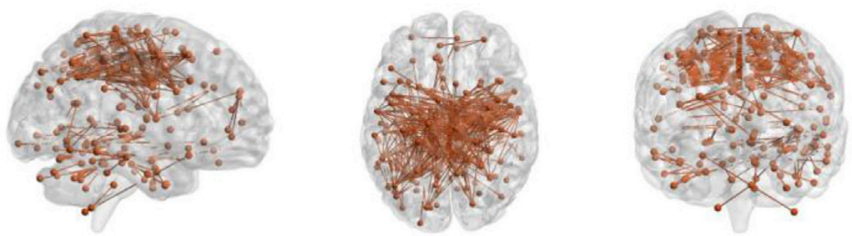

Fig. 3. With respect to ROI connections associated with high IRV (i.e., poor sustained attention), the ADHD symptom exhibited significantly stronger connectivity between ROIs, compared to controls.

attention tasks (Brisenden et al., 2016). In a large healthy sample $(\mathrm{N}=1000)$, intrinsic functional connectivity patterns were observed between lateral cerebellar areas of crus I/II with DMN, as well as between anterior Crus I with DLPFC, IPL, a pre-SMA midline border and ACC (Buckner et al., 2011). The finding indicates that Crus I/II are major cerebellar components of the DMN (Buckner et al., 2011). One study investigated functional connectivity of this DMN cerebellar component in healthy adults and adults with ADHD, finding that in the healthy sample, less anticorrelations between Crus I/II and DAN regions was associated with greater inattention (high IRV). Adding to this work, we found that anti-correlations between left-lateralized Crus I/II with right-lateralized frontoparietal regions predominantly characterize good sustained attention in our normative sample (Kucyi et al., 2015).

For average fMRI activation, high IRV was associated with activation in precentral and postcentral gyri bilaterally across all trials and in left SMA during Stop Success trials, a region responsible for successful stopping, monitoring and resolving task conflict (Verbruggen and Logan, 2008). High IRV was also associated with DMN, including Go-related activation in right precuneus and Stop-Success activation in precuneus bilaterally. DMN activation is thought to infringe upon neuronal circuits underlying task performance, and given that DMN deactivation is typically required for efficient sustained attentional processes (Kelly et al., 2008; Broyd et al., 2009), this positive IRV-DMN is unsurprising. However, DMN connectivity increases with maturation into young adulthood (Anderson et al., 2011; Fair et al., 2007b), suggesting that functional connectivity analyses may shed new light on such neural processes. With respect to the functional connectivity signature of high IRV, our findings describe robust positive bilateral connections within the motor network, and between motor with parietal and limbic networks. The top 10 nodes correlated with IRV were all positive interhemispheric connections within the motor network, characterizing poor sustained attention. The observed bilateral pattern of motor activation likely reflecting the task format, which required both left- and right-hand responses. Unlike Rosenberg and colleagues (Rosenberg et al., 2015), who found that poorer sustained attention in adults was related to connections between temporal and parietal networks and within the cerebellum, the observed positive motor-motor coupling may reflect a snapshot of neural development in early adolescence. For example, age-related decreases in motor connectivity have been observed in a large sample of healthy children and young adults (Van Duijvenvoorde et al., 2016). Our findings lend support for a more predominant functional segregation of neural networks in childhood and greater functional integration later on in adulthood (Konrad and Eickhoff, 2010).

Numerous studies have demonstrated behavioral and neural deficits in sustained attentional processes in ADHD (Kofler et al., 2013). Behaviorally, our results show that adolescents with ADHD symptoms have significantly increased IRV relative to controls, as previously demonstrated (Albaugh et al., 2017; Rubia et al., 2003). Consistent with this behavioral difference, the ADHD symptom group had significantly increased activation in the left postcentral gyrus during Stop Fail and Stop Success trials, compared to controls. This is similar to findings in 8-13 year-old children, whereby 25 children with ADHD had greater
IRV-related activation in left postcentral and right precentral gyri and IPL during Go trials on a Go/No-go task, as well as in prefrontal and parietal regions during No-go trials, compared to 25 controls (Suskauer et al., 2008). Similar patterns of motor connectivity dysfunction have also been shown in resting-state studies of children (McLeod et al., 2014) and young adults with ADHD (Kelly et al., 2017). Here, we found that, relative to asymptomatic controls, the ADHD symptom group had stronger positive connectivity within primarily motor networks whose connectivity was positively associated with ICV (i.e., poor sustained attention) in the normative group. The reason for the divergent results (weaker vs. stronger connectivity in motor networks in ADHD) likely reflects the fact that we measured functional connectivity during task performance, rather than at rest. Stronger task activation and stronger task-based functional connectivity may be necessary in the context of weaker baseline functional connectivity within motor networks, a hypothesis that could be tested by collecting both resting state and task data from a sample of adolescents with ADHD. Overall, our findings add to the growing body of literature linking dysfunctional premotor/motor systems to poorer behavioral control in ADHD (Suskauer et al., 2008). Adolescents with ADHD (Shaw et al., 2007) and subclinical attention deficits (Ducharme et al., 2012; Shaw et al., 2011) also display delays in cortical maturation, and as such, it is possible that any localized motor connectivity associated with IRV exhibited by the individuals within our samples will subside as more global, specialized brain networks develop.

At the other end of the spectrum of brain-behavior relationships with IRV, average fMRI activation revealed no differences for brain regions associated with low IRV between the ADHD symptom and control group. With respect to the functional connectivity signature of low IRV, no group differences were observed for functional connections associated with low IRV (i.e., good sustained attention). Studies have previously demonstrated that brain-behavior relationships can be modified in the presence of categorical diagnoses such as ADHD, and that not all diagnostic effects can be simplistically understood as dimensional (i.e., a continuum of too much or too little functional connectivity) (Chabernaud et al., 2012; Mennes et al., 2011). Our result may reflect a similarly complex interaction between the dimensional brain-behavior relationships associated with ADHD symptoms and IRV.

Data-driven analyses are commonly applied to resting-state data, whereas directional analyses (i.e., focusing on particular seed regions) are usually used in task-based studies (Ernst et al., 2015). Our findings solidify the importance of data-driven functional connectivity analyses, rather than constraining ROIs a priori (Turk-Browne, 2013) in order to better characterize cognitive processes. Although the brain-behavioral findings for sustained attention are reliable, there are some caveats to this study. The relationship between IRV and age may be a sensitive marker of neural development (Tamnes et al., 2012) and considering the major brain changes that occur in adolescence (Luciana, 2010), it is unclear if the observed functional trends reflect some sort of developmental delay or if they will persist as these adolescents develop. Secondly, although ADHD symptomatology revealed attentional anomalies in behavior and brain, our subclinical sample-size was small, and further investigations of ADHD symptomatology using larger datasets will be required. Thirdly, the ability to rigorously measure fluctuations in temporal resolution, combined with the corresponding physiological responses (head motion, respiration) remains a challenge (Poldrack, 2015; Kuntsi and Klein, 2011). The influence of head motion on functional connectivity for example is a well-documented issue, and is particularly troublesome for ADHD-related analyses. Prominent global artifacts tend to be present in scans and current methods do not adequately target these artifacts for removal (Power et al., 2017a). Broadly speaking, nuisance regression is the dominant approach for removing signal confounds, although it increases the risk of reducing signals of interest (Caballero-Gaudes and Reynolds, 2017). Scrubbing procedures can alter the temporal structure of timeseries data (Yan et al., 2013), therefore it was not implemented in this case. Some previous work indicates functional connectivity patterns remain largely unchanged after scrubbing, and that 
including mean framewise displacement as a group-level covariate yields similar results to scrubbing (Yan et al., 2013; Di Martino et al., 2014; Fair et al., 2013). The issue of head motion was at least partially addressed here by considering motion parameters as covariates in the statistical analysis (Yan et al., 2013). Global signal regression was also used in the current analyses, given that several reports have indicated its merits in robustly handling in-scanner movement (see (Ciric et al., 2017; Power et al., 2017b; Burgess et al., 2016)). Nevertheless, we found head motion (i.e., mean framewise displacement) significantly correlated with IRV in the large normative sample ( $\mathrm{n}=758 ; r=0.22)$, but not in the smaller ADHD symptom and control samples (each $\mathrm{n}=30$ ) similar to previous research (Rosenberg et al., 2015). This further highlights the importance of large sample sizes in order to control for spurious effects on functional connectivity data.

\section{Conclusion}

The current findings serve to advance our understanding of the brain networks associated with sustained attentional processes. Functional connectivity between a global array of networks, including the cerebellum, and motor, prefrontal and occipital cortices serve as a robust indicator for sustained attention. In particular, specific subregions of the cerebellum Crus I/II are robustly linked to sustained attention, while the involvement of motor connectivity in both low and high attention networks highlights its significant role in adolescent attention and cognition. In addition, the current research suggests that fMRI activation and functional connectivity within the motor network in the absence of higher order cognitive networks, may constitute a novel indicator of low sustained attention. The findings provide a solid basis for further research of cerebellar connectivity with motor networks in sustained attention. One future direction will also be to examine the extent to which these networks will predict subsequent inattention trajectories into adulthood.

\section{Disclosures}

Dr. Banaschewski has served as an advisor or consultant to BristolMyers Squibb, Desitin Arzneimittel, Eli Lilly, Medice, Novartis, Pfizer, Shire, UCB, and Vifor Pharma; he has received attendance support, conference support, or speaking fees from Eli Lilly, Janssen McNeil, Medice, Novartis, Shire, and UCB; and he is involved in clinical trials conducted by Eli Lilly, Novartis, and Shire; the present work is unrelated to these relationships. The other authors report no biomedical financial interests or potential conflicts of interest.

\section{Acknowledgments}

This work received support from the following sources: L. O'Halloran, L. Jollans and K. Ruddy are supported by The Irish Research Council (GOIPG/2016/1635; GOIPG/2014/418; GOIPD/2017/798). Z. Cao is supported by China Scholarship Council. R. Whelan is supported by Science Foundation Ireland (16/ERCD/3797) and a Brain and Behavior Research Foundation Young Investigator award (\#23599). C. Kelly is supported by the National Institute of Mental Health (R03MH104334, PI: Kelly) and a Brain and Behavior Research Foundation Young Investigator award (\#24065). This work received support from the following sources: the European Union-funded FP6 Integrated Project IMAGEN (Reinforcement-related behavior in normal brain function and psychopathology) (LSHM-CT- 2007-037286), the Horizon 2020 funded ERC Advanced Grant 'STRATIFY' (Brain network based stratification of reinforcementrelated disorders) (695313), ERANID (Understanding the Interplay between Cultural, Biological and Subjective Factors in Drug Use Pathways) (PR-ST-0416-10004), BRIDGET (JPND: BRain Imaging, cognition Dementia and next generation GEnomics) (MR/N027558/1), the FP7 projects IMAGEMEND(602450; IMAging GEnetics for MENtal Disorders) and MATRICS (603016), the Innovative Medicine Initiative Project EU-
AIMS (115300-2), the Medical Research Council Grant 'c-VEDA' (Consortium on Vulnerability to Externalizing Disorders and Addictions) (MR/N000390/1), the Swedish Research Council Formas, the Medical Research Council, the National Institute for Health Research (NIHR) Biomedical Research Centre at South London and Maudsley NHS Foundation Trust and King's College London, the Bundesministeriumfür Bildung und Forschung (BMBF grants 01GS08152; 01EV0711; eMED SysAlc01ZX1311A; Forschungsnetz AERIAL), the Deutsche Forschungsgemeinschaft (DFG grants SM 80/7-1, SM 80/7-2, SFB 940/1). Further support was provided by grants from: ANR (project AF12NEUR0008-01 - WM2NA, and ANR-12-SAMA-0004), the Fondation de France, the Fondation pour la Recherche Médicale, the Mission Interministérielle de Lutte-contre-les-Drogues-et-les-Conduites-Addictives (MILDECA), the Fondation pour la Recherche Médicale (DPA20140629802), the Fondation de l'Avenir, Paris-Sud University IDEX 2012; the National Institutes of Health, U.S.A. (Axon, Testosterone and Mental Health during Adolescence; RO1 MH085772-01A1), and by NIH Consortium grant U54 EB020403, supported by a cross-NIH alliance that funds Big Data to Knowledge Centres of Excellence.

\section{Appendix A. Supplementary data}

Supplementary data related to this article can be found at https://doi. org/10.1016/j.neuroimage.2017.12.030.

\section{References}

Albaugh, M.D., Orr, C., Chaarani, B., Althoff, R.R., Allgaier, N., D'Alberto, Hudson, K., Mackey, S., Spechler, P., Banaschewski, T., Brühl, R., Bokde, A., Bromberg, U., Büchel, C., Cattrell, A., Conrod, P., Desrivières, S., Flor, H., Frouin, V., Gallinat, J. Goodman, R., Gowland, P., Grimmer, Y., Heinz, A., Kappel, V., Martinot, J., Martinot, M., Nees, F., Orfanos, D., Penttilä, J., Poustka, L., Paus, T., Smolka, M., Struve, M., Walter, H., Whelan, R., Schumann, G., Garavan, H., Potter, A., 2017. Inattention and reaction time variability are linked to ventromedial prefrontal volume in adolescents. Biol. Psychiatr. http://doi.org/10.1016/j.biopsych.2017.01. 003.

Anderson, J.S., Ferguson, M.A., Lopez-Larson, M., Yurgelun-Todd, D., 2011. Connectivity gradients between the default mode and attention control networks. Brain Connect. 1 (2), 147-157.

Balota, D.A., Tse, C.S., Hutchison, K.A., Spieler, D.H., Duchek, J.M., Morris, J.C., 2010. Predicting conversion to dementia of the Alzheimer's type in a healthy control sample: the power of errors in stroop color naming. Psychol. Aging 25 (1), 208. https://doi.org/10.1037/a0017474.

Barber, A.D., Jacobson, L.A., Wexler, J.L., Nebel, M.B., Caffo, B.S., Pekar, J.J., Mostofsky, S.H., 2015. Connectivity supporting attention in children with attention deficit hyperactivity disorder. Neurol. Clin. 7, 68-81.

Barkley, R.A., 1997. Behavioral inhibition, sustained attention, and executive functions: constructing a unifying theory of ADHD. Psychol. Bull. 121 (1), 65.

Bellgrove, M.A., Hester, R., Garavan, H., 2004. The functional neuroanatomical correlates of response variability: evidence from a response inhibition task. Neuropsychologia 42 (14), 1910-1916.

Bellgrove, M.A., Hawi, Z., Kirley, A., Gill, M., Robertson, I.H., 2005. Dissecting the attention deficit hyperactivity disorder (ADHD) phenotype: sustained attention, response variability and spatial attentional asymmetries in relation to dopamine transporter (DAT1) genotype. Neuropsychologia 43 (13), 1847-1857.

Böttger, J., Schäfer, A., Lohmann, G., Villringer, A., Margulies, D.S., 2014. Threedimensional mean-shift edge bundling for the visualization of functional connectivity in the brain. IEEE Trans. Visual. Comput. Graph. 20 (3), 471-480.

Brisenden, J.A., Levin, E.J., Osher, D.E., Halko, M.A., Somers, D.C., 2016. Functional evidence for a cerebellar node of the dorsal attention network. J. Neurosci. 36 (22), 6083-6096.

Broyd, S.J., Demanuele, C., Debener, S., Helps, S.K., James, C.J., Sonuga-Barke, E.J., 2009. Default-mode brain dysfunction in mental disorders: a systematic review. Neurosci. Biobehav. Rev. 33, 279-296.

Buckner, R.L., 2013. The cerebellum and cognitive function: 25 years of insight from anatomy and neuroimaging. Neuron 80 (3), 807-815.

Buckner, R.L., Krienen, F.M., Castellanos, A., Diaz, J.C., Yeo, B.T., 2011. The organization of the human cerebellum estimated by intrinsic functional connectivity. J. Neurophysiol. 106, 2322-2345. https://doi.org/10.1152/jn.00339.2011.

Burgess, G.C., Kandala, S., Nolan, D., Laumann, T.O., Power, J.D., Adeyemo, B., Harms, M.P., Petersen, S.E., Barch, D.M., 2016 Nov 1. Evaluation of denoising strategies to address motion-correlated artifacts in resting-state functional magnetic resonance imaging data from the human connectome project. Brain Connect. 6 (9), 669-680.

Caballero-Gaudes, César, Reynolds, Richard C., 2017. Methods for cleaning the BOLD fMRI signal. Neuroimage 128-149.

Cai, W., Ryali, S., Chen, T., Li, C.S.R., Menon, V., 2014. Dissociable roles of right inferior frontal cortex and anterior insula in inhibitory control: evidence from intrinsic and 
task-related functional parcellation, connectivity, and response profile analyses across multiple datasets. J. Neurosci. 34 (44), 14652-14667.

Castellanos, F.X., Aoki, Y., 2016. Intrinsic functional connectivity in attention-deficit/ hyperactivity disorder: a science in development. Biol. Psychiatr.: cognitive neuroscience and neuroimaging 1 (3), 253-261.

Castellanos, F.X., Sonuga-Barke, E.J., Scheres, A., Di Martino, A., Hyde, C., Walters, J.R. 2005. Varieties of attention-deficit/hyperactivity disorder-related intra-individual variability. Biol. Psychiatr. 57 (11), 1416-1423. http://doi.org/10.1016/j.biopsych. 2004.12.005.

Castellanos, F.X., Sonuga-Barke, E.J., Milham, M.P., Tannock, R., 2006. Characterizing cognition in ADHD: beyond executive dysfunction. Trends Cognit. Sci. 10 (3), 117-123. https://doi.org/10.1016/j.tics.2006.01.011.

Castellanos, F.X., Margulies, D.S., Kelly, C., Uddin, L.Q., Ghaffari, M., Kirsch, A., Shaw, D. Shehzad, Z., Di Martino, A., Biswal, B., Sonuga-Barke, E.J., Rotrosenc, J., Adlerc, L., Milhama, M., 2008. Cingulate-precuneus interactions: a new locus of dysfunction in adult attention-deficit/hyperactivity disorder. Biol. Psychiatr. 63 (3), 332-337. http://doi.org/10.1016/j.biopsych.2007.06.025.

Castellanos, F.X., Kelly, C., Milham, M.P., 2009. The restless brain: attention-deficit hyperactivity disorder, resting - state functional connectivity, and intrasubject variability. Can. J. Psychiatr. 54 (10), 665-672.

Chabernaud, C., Mennes, M., Kelly, C., Nooner, K., Di Martino, A., Castellanos, F.X., Milham, M.P., 2012. Dimensional brain-behavior relationships in children with attention-deficit/hyperactivity disorder. Biol. Psychiatr. 71 (5), 434-442. http://doi. org/10.1016/j.biopsych.2011.08.013.

Cherbuin, N., Sachdev, P., Anstey, K.J., 2010. Neuropsychological predictors of transition from healthy cognitive aging to mild cognitive impairment: the PATH through Life Study. Am. J. Geriatr. Psychiatr. 18 (8), 723-733. https://doi.org/10.1097/ Jgp.0b013e3181cdecf1.

Chun, M.M., Golomb, J.D., Turk-Browne, N.B., 2011. A taxonomy of external and internal attention. Annu. Rev. Psychol. 62, 73-101.

Ciric, R., Wolf, D.H., Power, J.D., Roalf, D.R., Baum, G.L., Ruparel, K., Shinohara, R.T., Elliott, M.A., Eickhoff, S.B., Davatzikos, C., Gur, R.C., Bassett, D.S., Satterthwaite, T.D., 2017 Mar 14. Benchmarking of participant-level confound regression strategies for the control of motion artifact in studies of functional connectivity. Neuroimage 154 (1), 174-187. https://doi.org/10.1016/j.neuroimage. 2017.03.020

Corbetta, M., Shulman, G.L., 2002. Control of goal-directed and stimulus-driven attention in the brain. Nat. Rev. Neurosci. 3 (3), 201.

Dale, A.M., 1999. Optimal experimental design for event-related fMRI. Hum. Brain Mapp. 8 (2-3), 109-114.

Di Martino, A., Yan, C.G., Li, Q., Denio, E., Castellanos, F.X., Alaerts, K., Anderson, J.S. Assaf, M., Bookheimer, S.Y., Dapretto, M., Deen, B., 2014 Jun 1. The autism brain imaging data exchange: towards a large-scale evaluation of the intrinsic brain architecture in autism. Mol. Psychiatr. 19 (6), 659-667.

Dixon, R.A., Garrett, D.D., Lentz, T.L., MacDonald, S.W., Strauss, E., Hultsch, D.F., 2007 Neurocognitive markers of cognitive impairment: exploring the roles of speed and inconsistency. Neuropsychology 21 (3), 381. https://doi.org/10.1037/08944105.21.3.381.

Ducharme, S., Hudziak, J.J., Botteron, K.N., Albaugh, M.D., Nguyen, T.V., Karama, S. Evans, A.C., Brain Development Cooperative Group, 2012. Decreased regional cortical thickness and thinning rate are associated with inattention symptoms in healthy children. J. Am. Acad. Child Adolesc. Psychiatr. 51 (1), 18-27.

Eklund, A., Nichols, T.E., Knutsson, H., 2017. Cluster failure: why fMRI inferences for spatial extent have inflated false-positive rates. Proc. Natl. Acad. Sci. 113 (28), 7900-7905. https://doi.org/10.1073/pnas.1602413113.

Ernst, M., Torrisi, S., Balderston, N., Grillon, C., Hale, E.A., 2015. fMRI functional connectivity applied to adolescent neurodevelopment. Annu. Rev. Clin. Psychol. 11, 361-377.

Esterman, M., Noonan, S.K., Rosenberg, M., DeGutis, J., 2012. In the zone or zoning out? Tracking behavioral and neural fluctuations during sustained attention. Cerebr. Cortex 23 (11), 2712-2723. https://doi.org/10.1093/cercor/bhs261.

Esterman, M., Thai, M., Okabe, H., DeGutis, J., Saad, E., Laganiere, S.E., Halko, M.A. 2017. Network-targeted cerebellar transcranial magnetic stimulation improves attentional control. Neuroimage 156, 190-198.

Fair, D.A., Schlaggar, B.L., Cohen, A.L., Miezin, F.M., Dosenbach, N.U., Wenger, K.K., Fox, M.D., Snyder, A.Z., Raichle, M.E., Petersen, S.E., 2007. A method for using blocked and event-related fMRI data to study "resting state" functional connectivity. Neuroimage 35 (1), 396-405.

Fair, D.A., Dosenbach, N.U., Church, J.A., Cohen, A.L., Brahmbhatt, S., Miezin, F.M., Barch, D.M., Raichle, M.E., Petersen, S.E., Schlaggar, B.L., 2007. Development of distinct control networks through segregation and integration. Proc. Natl. Acad. Sci. 104 (33), 13507-13512.

Fair, D., Nigg, J.T., Iyer, S., Bathula, D., Mills, K.L., Dosenbach, N.U., Schlaggar, B.L., Mennes, M., Gutman, D., Bangaru, S., Buitelaar, J.K., 2013 Feb 4. Distinct neural signatures detected for ADHD subtypes after controlling for micro-movements in resting state functional connectivity MRI data. Front. Syst. Neurosci. 6, 80.

Fortenbaugh, F.C., DeGutis, J., Esterman, M., 2017. Recent theoretical, neural, and clinical advances in sustained attention research. Ann. N. Y. Acad. Sci. 1396, 70-91. https://doi.org/10.1111/nyas.13318.

Fox, M.D., Snyder, A.Z., Vincent, J.L., Corbetta, M., Van Essen, D.C., Raichle, M.E., 2005 The human brain is intrinsically organized into dynamic, anticorrelated functional networks. Proc. Natl. Acad. Sci. U. S. Am. 102 (27), 9673-9678.

Fox, M.D., Zhang, D., Snyder, A.Z., Raichle, M.E., 2009. The global signal and observed anticorrelated resting state brain networks. J. Neurophysiol. 101 (6), 3270-3283.

Garrison, K.A., Scheinost, D., Finn, E.S., Shen, X., Constable, R.T., 2015. The (in) stability of functional brain network measures across thresholds. Neuroimage 118, 651-661.
Glickstein, M., 2007. What does the cerebellum really do? Curr. Biol. 17 (19), R824-R827.

Goodman, R., Ford, T., Richards, H., Gatward, R., Meltzer, H., 2000. The development and well-being assessment: description and initial validation of an integrated assessment of child and adolescent psychopathology. J. Comp. Physiol. Psychol. 41 (5), 645-655.

Habas, C., Kamdar, N., Nguyen, D., Prater, K., Beckmann, C.F., Menon, V., Greicius, M.D., 2009. Distinct cerebellar contributions to intrinsic connectivity networks. J. Neurosci. 29 (26), 8586-8594.

Haynes, B.I., Bauermeister, S., Bunce, D., 2017. A systematic review of longitudinal associations between reaction time intraindividual variability and age-related cognitive decline or impairment, dementia, and mortality. J. Int. Neuropsychol. Soc. 23 (5), 431-445.

Hudziak, J.J., Achenbach, T.M., Althoff, R.R., Pine, D.S., 2007. A dimensional approach to developmental psychopathology. Int. J. Meth. Psychiatr. Res. 16 (S1).

Hultsch, D.F., MacDonald, S.W., Dixon, R.A., 2002. Variability in reaction time performance of younger and older adults. J. Gerontol. B Psychol. Sci. Soc. Sci. 57 (2), P101-P115.

Johnson, B.P., Pinar, A., Fornito, A., Nandam, L.S., Hester, R., Bellgrove, M.A., 2015. Left anterior cingulate activity predicts intra-individual reaction time variability in healthy adults. Neuropsychologia 72, 22-26. http://doi.org/10.1016/j. neuropsychologia.2015.03.015.

Kelly, A.C., Uddin, L.Q., Biswal, B.B., Castellanos, F.X., Milham, M.P., 2008. Competition between functional brain networks mediates behavioral variability. Neuroimage 39 (1), 527-537.

Kelly, C., Castellanos, F.X., Tomaselli, O., Lisdahl, K., Tamm, L., Jernigan, T., Newman, E., Epstein, J.N., Molina, B.S., Greenhill, L.L., Potkin, S.G., 2017. Distinct effects of childhood ADHD and cannabis use on brain functional architecture in young adults. Neuroimage: Clin. 13, 188-200. http://doi.org/10.1016/j.nicl.2016.09.012.

Kessler, D., Angstadt, M., Sripada, C., 2016. Growth charting of brain connectivity networks and the identification of attention impairment in youth. JAMA psychiatry 73 (5), 481-489. https://doi.org/10.1001/jamapsychiatry.2016.0088 pmid: 27076193.

Klein, C., Wendling, K., Huettner, P., Ruder, H., Peper, M., 2006. Intra-subject variability in attention-deficit hyperactivity disorder. Biol. Psychiatr. 60 (10), 1088-1097.

Kofler, M.J., Rapport, M.D., Sarver, D.E., Raiker, J.S., Orban, S.A., Friedman, L.M., Kolomeyer, E.G., 2013. Reaction time variability in ADHD: a meta-analytic review of 319 studies. Clin. Psychol. Rev. 33 (6), 795-811. http://doi.org/10.1016/j.cpr.2013. 06.001 .

Konrad, K., Eickhoff, S.B., 2010. Is the ADHD brain wired differently? A review on structural and functional connectivity in attention deficit hyperactivity disorder. Hum. Brain Mapp. 31 (6), 904-916.

Kucyi, A., Hove, M.J., Biederman, J., Van Dijk, K.R., Valera, E.M., 2015. Disrupted functional connectivity of cerebellar default network areas in attention-deficit/ hyperactivity disorder. Hum. Brain Mapp. 36 (9), 3373-3386.

Kuntsi, J., Klein, C., 2011. Intraindividual variability in ADHD and its implications for research of causal links. In: Behavioral Neuroscience of Attention Deficit Hyperactivity Disorder and its Treatment. Springer Berlin Heidelberg, pp. 67-91.

Langley, K., Heron, J., Smith, G.D., Thapar, A., 2012. Maternal and paternal smoking during pregnancy and risk of ADHD symptoms in offspring: testing for intrauterine effects. Am. J. Epidemiol. 176 (3), 261-268.

Langner, R., Eickhoff, S.B., 2013. Sustaining Attention to Simple Tasks: a Meta-analytic Review of the Neural Mechanisms of Vigilant Attention.

Leth-Steensen, C., Elbaz, Z.K., Douglas, V.I., 2000. Mean response times, variability, and skew in the responding of ADHD children: a response time distributional approach. Acta Psychol. 104 (2), 167-190.

Luciana, M., 2010. Adolescent Brain Development: Current Themes and Future Directions: Introduction to the Special Issue.

McLeod, K.R., Langevin, L.M., Goodyear, B.G., Dewey, D., 2014. Functional connectivity of neural motor networks is disrupted in children with developmental coordination disorder and attention-deficit/hyperactivity disorder. Neuroimage: Clin. 4, 566-575.

Mennes, M., Potler, N.V., Kelly, C., Di Martino, A., Castellanos, F.X., Milham, M.P., 2011. Resting state functional connectivity correlates of inhibitory control in children with attention-deficit/hyperactivity disorder. Front. Psychiatr. 2. http://doi.org/10.3389/ fpsyt.2011.00083.

Mullins, C., Bellgrove, M.A., Gill, M., Robertson, I.H., 2005. Variability in time reproduction: difference in ADHD combined and inattentive subtypes. J. Am. Acad. Child Adolesc. Psychiatr. 44 (2), 169-176. https://doi.org/10.1097/ 00004583-200502000-00009.

Namkung, H., Kim, S.H., Sawa, A., 2017. The insula: an underestimated brain area in clinical neuroscience, psychiatry, and neurology. Trends Neurosci. 40 (4), 200-207. https://doi.org/10.1016/j.tins.2017.02.002.

O'Halloran, L., Nymberg, C., Jollans, L., Garavan, H., Whelan, R., 2017. The potential of neuroimaging for identifying predictors of adolescent alcohol use initiation and misuse. Addiction 112 (4), 719-726.

Petersen, S.E., Posner, M.I., 2012. The attention system of the human brain: 20 years after. Annu. Rev. Neurosci. 35, 73-89.

Petersen, A.C., Crockett, L., Richards, M., Boxer, A., 1988. A self-report measure of pubertal status: reliability, validity, and initial norms. J. Youth Adolesc. 17 (2), 117-133. https://doi.org/10.1007/BF01537962.

Poldrack, R.A., 2015. Is "efficiency" a useful concept in cognitive neuroscience? Developmental cognitive neuroscience 11, 12-17.

Power, J.D., Mitra, A., Laumann, T.O., Snyder, A.Z., Schlaggar, B.L., Petersen, S.E., 2014. Methods to detect, characterize, and remove motion artifact in resting state fMRI. Neuroimage 84, 320-341. 
Power, J.D., Plitt, M., Laumann, T.O., Martin, A., 2017. Sources and implications of whole-brain fMRI signals in humans. Neuroimage 146, 609-625.

Power, J.D., Laumann, T.O., Plitt, M., Martin, A., Petersen, S.E., 2017 Sep 19. On global fMRI signals and simulations. Trends Cognit. Sci. 21 (12), 911-913. https://doi.org/ 10.1016/j.tics.2017.09.002.

Rosenberg, M.D., Finn, E.S., Scheinost, D., Papademetris, X., Shen, X., Constable, R.T., Chun, M.M., 2015. A neuromarker of sustained attention from whole-brain functional connectivity. Nat. Neurosci. 19, 165-171. https://doi.org/doi:10.1038/nn.4179.

Rosenberg, M.D., Finn, E.S., Scheinost, D., Constable, R.T., Chun, M.M., 2017. Characterizing attention with predictive network models. Trends Cognit. Sci. 21 (4), 290-302. http://doi.org/10.1016/j.tics.2017.01.011.

Rubia, K., Noorloos, J., Smith, A., Gunning, B., Sergeant, J., 2003. Motor timing deficits in community and clinical boys with hyperactive behavior: the effect of methylphenidate on motor timing. J. Abnorm. Child Psychol. 31 (3), 301-313.

Rubia, K., Smith, A.B., Taylor, E., Brammer, M., 2007. Linear age-correlated functional development of right inferior fronto-striato-cerebellar networks during response inhibition and anterior cingulate during error-related processes. Hum. Brain Mapp. 28 (11), 1163-1177.

Russell, V.A., Oades, R.D., Tannock, R., Killeen, P.R., Auerbach, J.G., Johansen, E.B., Sagvolden, T., 2006. Response variability in attention-deficit/hyperactivity disorder: a neuronal and glial energetics hypothesis. Behav. Brain Funct. 2 (1), 30. https:// doi.org/10.1186/1744-9081-2-30.

Saad, Z.S., Gotts, S.J., Murphy, K., Chen, G., Jo, H.J., Martin, A., Cox, R.W., 2012. Trouble at rest: how correlation patterns and group differences become distorted after global signal regression. Brain Connect. 2 (1), 25-32.

Salum, G.A., Sonuga-Barke, E., Sergeant, J., Vandekerckhove, J., Gadelha, A. Moriyama, T.S., Graeff-Martins, A.S., Manfro, G.G., Polanczyk, G., Rohde, L.A.P., 2014. Mechanisms underpinning inattention and hyperactivity: neurocognitive support for ADHD dimensionality. Psychol. Med. 44 (15), 3189-3201. https://doi. org/10.1017/S0033291714000919.

Scheinost, D., Benjamin, J., Lacadie, C.M., Vohr, B., Schneider, K.C., Ment, L.R., Papademetris, X., Constable, R.T., 2012. The intrinsic connectivity distribution: a novel contrast measure reflecting voxel level functional connectivity. Neuroimage 62 (3), 1510-1519.

Schumann, G., Loth, E., Banaschewski, T., Barbot, A., Barker, G., Büchel, C., Conrod, P.J., Dalley, J.W., Flor, H., Gallinat, J., Garavan, H., Heinz, A., Itterman, B., Lathrop, M., Mallik, C., Mann, K., Martinot, J., Paus, T., Poline, J.-B., Robbins, T.W., Rietschel, M., Reed, L., Smolka, M., Spanagel, R., Speiser, C., Stephens, D.N., Strohle, A., Struve, M., 2010. The IMAGEN study: reinforcement-related behaviour in normal brain function and psychopathology. Mol. Psychiatr. 15 (12), 1128-1139. https://doi.org/10.1038/ mp.2010.4.

Seeley, W.W., Menon, V., Schatzberg, A.F., Keller, J., Glover, G.H., Kenna, H., Reiss, A.L., Greicius, M.D., 2007. Dissociable intrinsic connectivity networks for salience processing and executive control. J. Neurosci. 27 (9), 2349-2356.

Shaw, P., Eckstrand, K., Sharp, W., Blumenthal, J., Lerch, J.P., Greenstein, D.E.E.A., Clasen, L., Evans, A., Giedd, J., Rapoport, J.L., 2007. Attention-deficit/hyperactivity disorder is characterized by a delay in cortical maturation. Proc. Natl. Acad. Sci. Unit. States Am. 104 (49), 19649-19654.

Shaw, P., Gilliam, M., Liverpool, M., Weddle, C., Malek, M., Sharp, W., Greenstein, D., Evans, A., Rapoport, J., Giedd, J., 2011. Cortical development in typically developing children with symptoms of hyperactivity and impulsivity: support for a dimensional view of attention deficit hyperactivity disorder. Am. J. Psychiatr. 168 (2), 143-151.

Shen, X., Tokoglu, F., Papademetris, X., Constable, R.T., 2013. Groupwise whole-brain parcellation from resting-state fMRI data for network node identification. Neuroimage 82, 403-415.

Shen, X., Finn, E.S., Scheinost, D., Rosenberg, M.D., Chun, M.M., Papademetris, X., Constable, R.T., 2017. Using connectome-based predictive modeling to predict individual behavior from brain connectivity. Nat. Protoc. 12 (3), 506-518.

Simmonds, D.J., Fotedar, S.G., Suskauer, S.J., Pekar, J.J., Denckla, M.B., Mostofsky, S.H., 2007. Functional brain correlates of response time variability in children. Neuropsychologia 45, 2147-2157. http://doi.org/10.1016/j.neuropsychologia.2007. 01.013.

Sonuga-Barke, E.J., Castellanos, F.X., 2007. Spontaneous attentional fluctuations in impaired states and pathological conditions: a neurobiological hypothesis. Neurosci. Biobehav. Rev. 31 (7), 977-986.

Stoodley, C.J., 2012. The cerebellum and cognition: evidence from functional imaging studies. Cerebellum 11 (2), 352-365.

Suskauer, S.J., Simmonds, D.J., Caffo, B.S., Denckla, M.B., Pekar, J.J., Mostofsky, S.H., 2008. fMRI of intrasubject variability in ADHD: anomalous premotor activity with prefrontal compensation. J. Am. Acad. Child Adolesc. Psychiatr. 47 (10), 1141-1150.

Szczepanski, S.M., Konen, C.S., Kastner, S., 2010. Mechanisms of spatial attention control in frontal and parietal cortex. J. Neurosci. 30 (1), 148-160.

Tamm, L., Narad, M.E., Antonini, T.N., O'Brien, K.M., Hawk, L.W., Epstein, J.N., 2012. Reaction time variability in ADHD: a review. Neurotherapeutics 9 (3), 500-508.

Tamnes, C.K., Fjell, A.M., Westlye, L.T., Østby, Y., Walhovd, K.B., 2012. Becoming consistent: developmental reductions in intraindividual variability in reaction time are related to white matter integrity. J. Neurosci. 32 (3), 972-982.

Turk-Browne, N.B., 2013. Functional interactions as big data in the human brain. Science 342 (6158), 580-584.

Van Duijvenvoorde, A.C.K., Achterberg, M., Braams, B.R., Peters, S., Crone, E.A., 2016 Testing a dual-systems model of adolescent brain development using resting-state connectivity analyses. Neuroimage 124, 409-420.

Vaurio, R.G., Simmonds, D.J., Mostofsky, S.H., 2009. Increased intra-individual reaction time variability in attention-deficit/hyperactivity disorder across response inhibition tasks with different cognitive demands. Neuropsychologia 47 (12), 2389-2396.

Verbruggen, F., Logan, G.D., 2008. Response inhibition in the stop-signal paradigm. Trends Cognit. Sci. 12 (11), 418-424.

Wager, T.D., Barrett, L.F., 2017. From Affect to Control: Functional Specialization of the Insula in Motivation and Regulation. bioRxiv, p. 102368.

Wechsler, D., 2003. Wechsler Intelligence Scale for Children-fourth Edition (WISC-iv) Technical and Interpretive Manual. Psychological Corporation, San Antonio, TX.

Woo, C.W., Krishnan, A., Wager, T.D., 2014. Cluster-extent based thresholding in fMRI analyses: pitfalls and recommendations. Neuroimage 91, 412-419.

Xia, M., Wang, J., He, Y., 2013. BrainNet Viewer: a network visualization tool for human brain connectomics. PLos One 8 (7) e68910.

Yan, C.G., Cheung, B., Kelly, C., Colcombe, S., Craddock, R.C., Di Martino, A., Li, Q., Zuo, X.N., Castellanos, F.X., Milham, M.P., 2013. A comprehensive assessment of regional variation in the impact of head micromovements on functional connectomics. Neuroimage 76, 183-201. 